Check for updates

Cite this: Chem. Soc. Rev., 2020, 49,6248

Received 7th January 2020

DOI: $10.1039 / \mathrm{d} 0 \mathrm{cs} 00009 \mathrm{~d}$

rsc.li/chem-soc-rev

\section{Chiral covalent organic frameworks: design, synthesis and property}

\author{
Xing Han, (D) $\dagger^{a}$ Chen Yuan, (D) $\dagger^{a}$ Bang Hou, ${ }^{a}$ Lujia Liu, (D) bc Haiyang Li, ${ }^{\text {d }}$ Yan Liu (D) *a \\ and Yong Cui (D)*a
}

Covalent organic frameworks (COFs) are constructed using reticular chemistry with the building blocks being connected via covalent bonds and have emerged as a new series of porous materials for multitudinous applications. Most COFs reported to date are achiral, and only a small fraction of COFs with chiral nature are reported. This review covers the recent advances in the field of chiral COFs (CCOFs), including their design principles and synthetic strategies, structural studies, and potential applications in asymmetric catalysis, enantioselective separation, and chiral recognition. Finally, we illustrate the remaining challenges and future opportunities in this field.

\section{Introduction}

Chirality is an important element in art, fashion, health, and manufacturing sectors, with broad applications ranging from fragrance, clinical analysis, to pharmaceutical, agrichemical, and other fine-chemical industries. ${ }^{1-5}$ For instance, chiral compounds

\footnotetext{
${ }^{a}$ School of Chemistry and Chemical Engineering, Frontiers Science Center for Transformative Molecules and State Key Laboratory of Metal Matrix Composites, Shanghai Jiao Tong University, Shanghai 200240, P. R. China.

E-mail:yongcui@sjtu.edu.cn,liuy@sjtu.edu.cn

${ }^{b}$ School of Chemical and Physical Sciences, Victoria University of Wellington, PO Box 600, Wellington 6140, New Zealand

${ }^{c}$ College of Biological, Chemical Science and Engineering, Jiaxing University, 118 Jiahang Road, Jiaxing 314001, China

${ }^{d}$ College of Chemistry and Molecular Engineering, Zhengzhou University,

Zhengzhou 450001, China

$\dagger \mathrm{X}$. H. and C. Y. contributed equally.
}

often exhibit distinct optical rotation or circular dichroism, and in many chiral pharmaceuticals, one of the enantiomers is often more active than the other enantiomer. ${ }^{6}$ In recent years, chiral science and technology, including synthesis, separation, structural analysis, and applications of chiral compounds, has been attracting increasing attention. ${ }^{7-9}$ There already exists a big market for chiral compounds, especially for chiral pharmaceuticals. According to a recent report by the United States Food and Drug Administration (FDA), 23 small molecule drugs were approved by FDA's Center for Drug Evaluation and Research (CDER) in 2019 alone, and 18 of them are chiral molecules. ${ }^{10}$

Porous solids are a class of materials with an increasing role in chiral science, technology, and industry. ${ }^{11,12}$ With decades of research and development, some key challenges have been identified. These include material synthesis and manufacturing, structure modification, establishing design principles for maximizing chiroptical activity, and efforts to broaden the scope

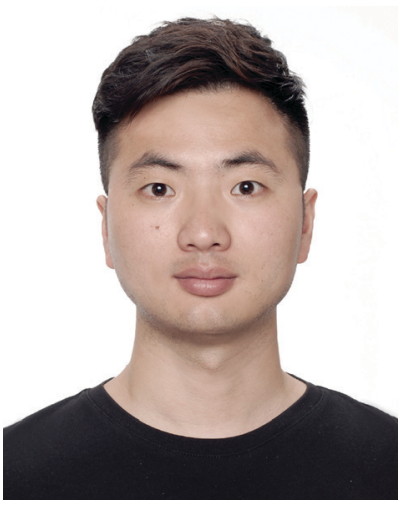

Xing Han
Xing Han was born in Anhui province, China, in 1990. He received his $B S$ degree from Anhui Normal University in 2013. Then, he joined the research group of Prof. Yong Cui at Shanghai Jiao Tong University and obtained his PhD degree in 2019 focusing on the design and synthesis of functional covalent organic frameworks.

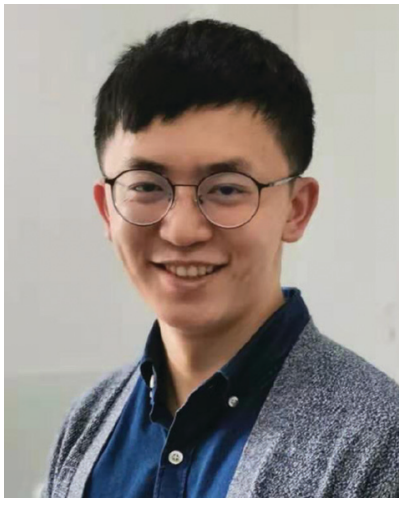

Chen Yuan was born in Yunnan province, China, in 1993. He received his $B S$ degree from Sichuan University in 2015. In the fall of 2015, he joined Prof. Yong Cui's group at Shanghai Jiao Tong University for a PhD degree. His current research interest focuses on chiral covalent organic frameworks for chiral recognition and separation.

\title{
Chen Yuan
}


of applications. ${ }^{13,14}$ To tackle these key challenges, new chiral crystalline porous solids have been designed and synthesized. ${ }^{14-16}$ These materials, such as MOFs, ZIFs, etc., feature unambiguous structures, homogeneous pores, and discrete catalytic sites. They can be rationally designed with specific structures and functions at the molecular level when some building block geometry and chemical compatibility arguments are considered. In addition, these materials exhibit excellent performance in asymmetric catalysis, enantioseparation, chiral recognition and chiral optics. ${ }^{15-18}$ Despite these merits, the stability of most crystalline porous solids still limits their wide utilization in chiral science and technology.

As a new type of porous material, covalent organic frameworks (COFs) have emerged as a novel platform for material design and functional explorations, with potential applications in diverse areas such as gas storage and separation, optoelectronics, energy storage, sensing, and catalysis. ${ }^{19-22}$ A judicious choice of building blocks can lead to porous COFs, with advantages such as highly ordered pore architectures, structural diversity, facile functionalization, high electrical conductivity, superior chemical stability and mechanical processability. ${ }^{23-27}$ These features warrant chiral COFs (CCOFs) with enormous potential in chiral recognition, chiral separation, asymmetric catalysis, and chiral optics. ${ }^{28-30}$

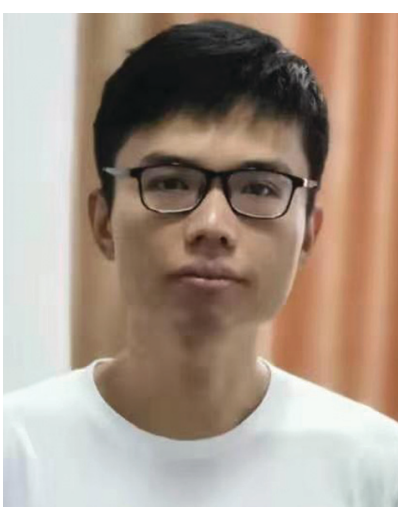

Bang Hou
Bang Hou was born in Henan province, China, in 1994. He received his $B S$ degree from China Agricultural University in 2015. In the fall of 2015, he joined Prof. Yong Cui's group at Shanghai Jiao Tong University for a PhD degree. His current research interest focuses on chiral covalent organic frameworks for catalysis and separation.

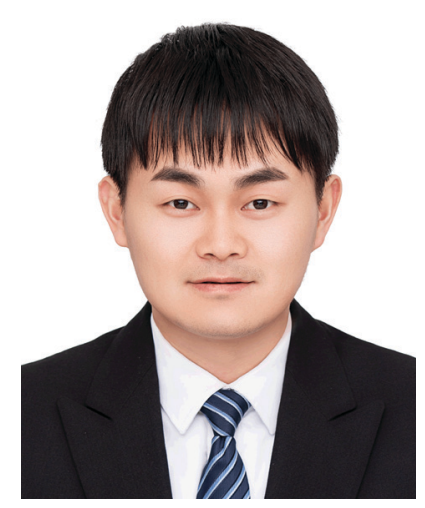

Haiyang Li
Haiyang Li was born in 1990. He obtained his BS in chemistry from Zhengzhou University in 2011, and received his $P h D$ degree under the supervision of Prof. Thomas C. W. Mak and Prof. Shuang-Quan Zang from Zhengzhou University in 2017. He joined the college of chemistry of Zhengzhou University in 2017. His research focuses on the design and synthesis of functional porous framework materials.

The development of CCOFs is of great significance to advancing chiral science. Nevertheless, a major challenge that remains in CCOFs is to balance the asymmetry of chiral monomers and material crystallinity. Therefore, the precise control of chirality, functionality, and crystallinity is a key endeavour. Only a small number of CCOFs have been reported so far, and their network topologies are very limited.

The research progress of COFs has been covered in several important reviews, ${ }^{20-22,31-41}$ with chiral COFs being only briefly mentioned. ${ }^{31,32,35-37}$ This review aims to provide a comprehensive overview of CCOFs with an emphasis on the design, synthesis, and applications in asymmetric catalysis, chiral separation, and chiral recognition. The review will also cover chiral COFs with some case studies to illustrate the design and synthetic strategies, structural feature and applications.

\section{Design and synthesis}

Since Yaghi and co-workers first reported COF powders using the solvothermal method in $2005,{ }^{19}$ more than 50 research

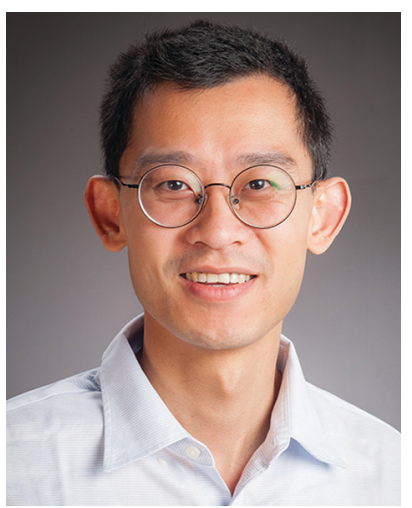

Lujia Liu
Lujia Liu obtained his BSc from Shanghai Jiao Tong University in 2009. After working at Nippon Paint (China) Co. Ltd as a formulation chemist, he moved to New Zealand and completed his PhD at Massey University in 2015. He then worked as a postdoctoral fellow at Northwestern University in the United States. In 2020, he joined Victoria University of Wellington, New Zealand, as a lecturer. He is the recipient of the 2015 Royal Society of New Zealand Hatherton Award. His current research interests lie in crystalline porous solids.

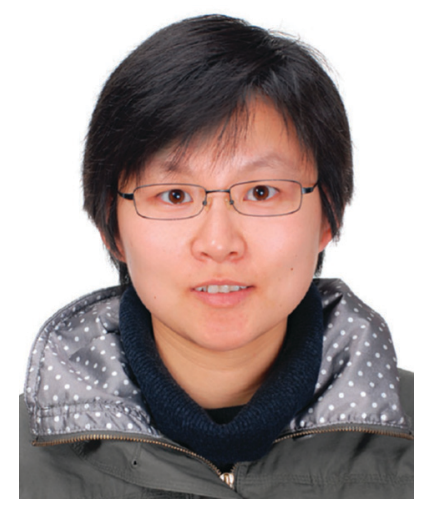

Yan Liu
Yan Liu received her BS degree in applied chemistry in 2006 and PhD in chemistry in 2011 from Shanghai Jiao Tong University. She conducted postdoctoral research at the University of North Carolina (2011-2013). She joined Shanghai Jiao Tong University in 2014, where she is now a professor of chemistry. Her research interest focuses on supramolecular chemistry and coordination chemistry. 
groups have diversified synthetic methods, including solvothermal, ${ }^{42-44}$ ionothermal, ${ }^{45-47}$ microwave, ${ }^{48,49}$ and roomtemperature methods. ${ }^{50-52}$

(1) Solvothermal method: similar to the synthesis of MOFs and zeolites, the solvothermal synthesis method is the most commonly used method to synthesize COFs, and it usually takes 1 to 9 days and requires heating in an inert gas atmosphere. $^{42-44}$ (2) Ionothermal synthesis of COFs was first reported by Thomas et al. in $2008 .^{45}$ The covalent triazine-based frameworks (CTFs) were synthesized by cyclotrimerisation of nitrile building units at $400{ }^{\circ} \mathrm{C}$ in molten $\mathrm{ZnCl}_{2}$. (3) Microwave assisted method: it was found that microwaves could be used to synthesize crystalline COF materials in $2009 .{ }^{48}$ Cooper and his colleagues synthesized COF-5 by microwave heating for $20 \mathrm{~min}$, which was 210 times faster than solvothermal synthesis. (4) Room-temperature synthesis was achieved in several approaches, including mechanochemical (MC) grinding and interfacial synthesis, with the latter taking place at the interface of two liquids at room temperature. Using these methods, abundant, robust, stable, crystalline and highly porous functional materials were reported. ${ }^{24,53-57}$

The crystallinity of COFs may be affected by a complex of factors. Temperature: Reaction temperature can affect the reaction output; ${ }^{19,58-61}$ solvent: the choice of the reaction solvent is a crucial factor because it controls the solubility of the monomers and influences the dynamics of covalent bond formation; ${ }^{62-64}$ catalyst: the type and amount of catalysts can influence the reaction rate. This is a key factor to synthesize crystalline COF powders. ${ }^{65-67}$ Moreover, the reaction rate influenced by the concentration of monomers is also a key factor to synthesize COF powders with structural regularity.

Although several COFs have recently been characterized by single-crystal X-ray diffraction, it remains a big challenge to produce single crystals of COFs. ${ }^{68,69}$ The crystalline structures of reported COFs have been most commonly determined by analysis of powder X-ray diffraction (PXRD) data and aided by consideration of geometry principles developed in reticular chemistry. ${ }^{19,70}$ Furthermore, the structure of COFs which have

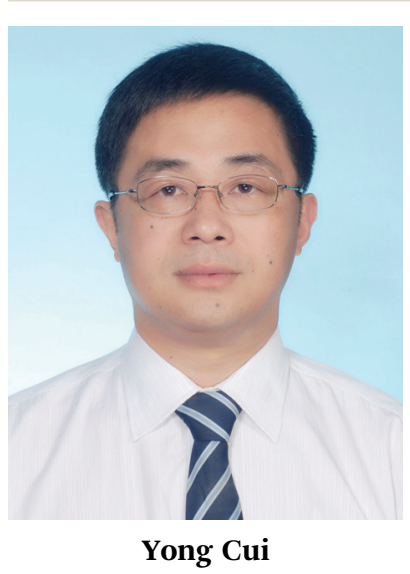

Yong Cui received his $P h D$ in physical chemistry in 1999 from Fujian Institute of Research on the Structure of Matter, CAS. He conducted postdoctoral research at the University of Science and Technology of China, University of North Carolina and University of Chicago from 1999 to 2005. He joined Shanghai Jiao Tong University in 2005, where he is now a chair professor of chemistry. His research interest focuses on the fields of molecular assembly, porous materials and chiral materials. high crystallinity also could be determined by 3D rotation electron diffraction. ${ }^{71}$

Despite these synthetic successes, chiral COFs were not reported until 2014. This is due in part to the low-symmetry nature of chiral monomers, which contradicts with the observations that most COFs crystallized in high-symmetry space groups with multiple crystallographic symmetry elements residing on each monomer. In addition, when using chiral monomers with specific functions, their solubility and chemical compatibility are also important considerations to obtain crystalline products. Up to now, all of the CCOFs have been synthesized by the solvothermal method.

The synthetic methods of CCOFs reported can also be classified into three strategies according to their synthetic pathways (Fig. 1)..$^{28,72-90}$

(1) Post-synthesis: synthesis of CCOFs from achiral parent COFs by post-synthetic modification of the organic framework.

(2) Direct synthesis: synthesis of a homochiral COF from enantiopure monomers, including chiral skeleton monomers and achiral skeleton monomers with appended chiral centres, as cross-linking building units.

(3) Chiral induction synthesis: synthesis of a homochiral COF from achiral organic precursors by chiral catalytic induction.

Here we briefly discuss the synthetic conditions and synthetic strategies of CCOF powders (Table 1). Based on the above synthetic methods, several different types of CCOFs have been reported. As shown in Fig. 2, there are CCOFs reported based on versatile monomers and chiral moieties, such as BINOL, TADDOL, salen, pyrrolidine, L-proline, L-imidazolidine and biomolecules.

\subsection{Post-synthesis}

The post-synthesis modification (PSM) strategy has been widely used in chiral porous solid materials fields. ${ }^{91-93}$ For example, recent studies demonstrated that organic linkers of MOFs are amenable to PSM with a variety of covalent transformations. ${ }^{94-96}$ These works suggest that it is feasible to synthesize COFs via the PSM process.

Indeed, several functional COFs with various applications were synthesized via PSM. ${ }^{32,97-99}$ Inspired by these works, researchers envisioned synthesizing CCOFs via PSM by introducing chiral moieties to the achiral COF skeletons. This strategy enables incorporation of chiral functionality that cannot be introduced directly (Fig. 1a).

The first CCOF synthesized via PSM is an imine-linked, porphyrin-based homochiral COF reported by Jiang et al. ${ }^{72}$ The parent COF features a $2 \mathrm{D}$ layered structure with a $1 \mathrm{D}$ tetragonal tubular channel. The linear monomer has allyl ether functional groups available for PSM. Through "clicking" $(S)$-pyrrolidine on the achiral COF skeleton, a homochiral COF is obtained with catalytic activity towards asymmetric Michael additions (Fig. 3). This example demonstrated the utility of PSM to synthesize catalytically active CCOFs from the parent, achiral COFs. Subsequently, in 2015, the Jiang group reported the synthesis of an achiral 2D hexagonal COF that is stable towards water, strong acids, and strong bases by 
a)

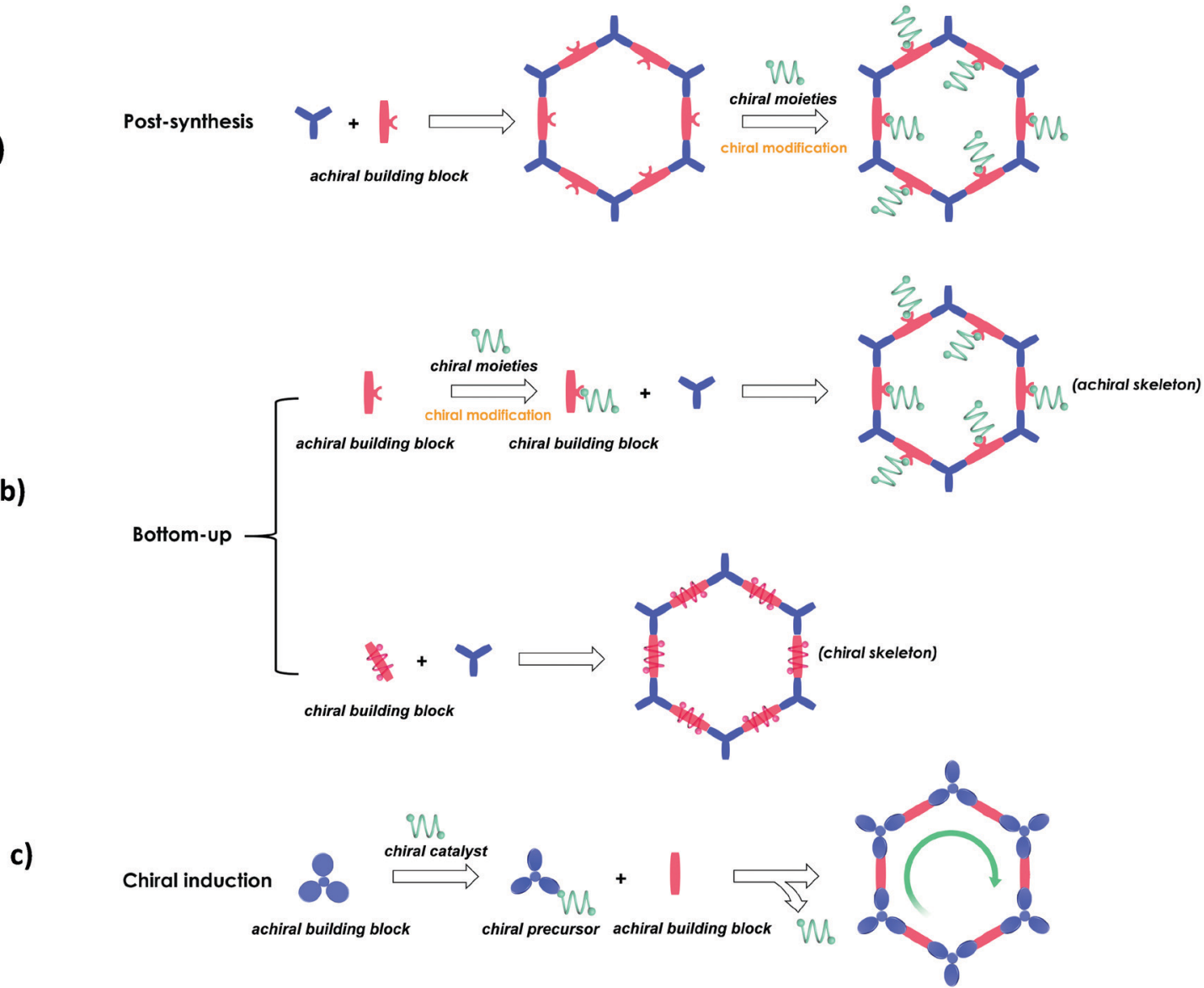

Fig. 1 General strategies for the design and synthesis of chiral COF materials.

incorporating methoxy groups onto the terephthalaldehyde monomer. ${ }^{73}$ After a quantitative azide-ethynyl click reaction, the achiral COF was converted to a catalytically active CCOF by anchoring (S)-pyrrolidine onto the channel walls via PSM, with both crystallinity and porosity retained (Fig. 3b).

Besides covalently binding small organic molecules as chiral appendants, in the past two years, an increasing number of CCOFs have been synthesized via immobilization and stabilization of natural biomolecules. Homochirality is observed in all life on Earth, and chiral biomolecules, such as amino acids, enzymes, and sugars, are ubiquitous. These homochiral systems are crucial for biological processes including molecular recognition, enzymatic function and information storage. ${ }^{100-102}$ Therefore, introducing chiral biomolecules into COFs provides a short-cut to constructing privileged functional CCOFs. The first successful example of PSM with natural biomolecules was reported in 2018 by Ma and Chen et al. The authors demonstrated a general approach by anchoring a series of biomolecules (lysozyme, tripeptides, and lysine) into a polyimide mesoporous achiral 2D COF (biomolecule $\subset \mathrm{COF} 1$ ) by covalent immobilization. This strategy introduces chirality into achiral COFs. ${ }^{74} \mathrm{~A}$ covalent coupling strategy was applied to immobilize biomolecules via a coupling reaction of $-\mathrm{NH}_{2}$ (from the biomolecules) and $-\mathrm{COOH}$ (from the COFs) (Fig. 4). The afforded lysozyme-immobilized COFs maintained more than $90 \%$ of enzymatic activity and showed excellent enzyme loading capacity. Notably, lysozyme immobilized in the COF does not leach out during the catalytic recycling process, according to NMR spectroscopy.

In the same year, Ma's group reported another example by soaking achiral 2D COFs in a phosphate buffer solution of a chiral enzyme, namely lipase PS, to form chiral biocomposites. ${ }^{77}$ The resulting composites have shown the ability to boost the stability and robustness of lipase PS. It is worth noting that functional groups on the COF pores could affect the compatibility between the enzyme and the achiral COFs.

Cyclodextrin (CD) is an important biomolecule with a fascinating intrinsically chiral cavity. ${ }^{78-80}$ Very recently, the Cui group reported the covalent-immobilization of $\beta-\mathrm{CD}$ in a $\mathrm{COF}^{81}$ They utilized a multivariate strategy to crystallize two vinyl-functionalized 2D COFs by imine condensation of a $C_{3}$-symmetric trialdehyde and a mixture of diamines with and without divinyl groups. Both multivariant COFs feature hexagonal 1D mesoporous channels formed by eclipsed or staggered stacking of layered hexagonal networks. PSM with chiral 6-deoxy-6-mercapto- $\beta$-cyclodextrin (SH- $\beta$-CD) via thiol-ene click reactions delivered CCOFs (CD-COF-1 and -2) with high chiral recognition performances (Fig. 5). $\mathrm{N}_{2}$ adsorption-desorption 
Table 1 Summary of the methods for the synthesis of CCOFs

\begin{tabular}{|c|c|c|}
\hline Methods & CCOFs & Conditions \\
\hline \multirow[t]{5}{*}{ Post-synthesis } & {$[\mathrm{Pyr}]_{x}-\mathrm{H} 2 \mathrm{P}-\mathrm{COFs}$} & $\begin{array}{l}\text { Synthesis by click reaction of achiral }[\mathrm{HC} \equiv \mathrm{C}]_{X}-\mathrm{H}_{2} \mathrm{P}-\mathrm{COFs} \text { and }(S) \text {-2-(azidomethyl)- } \\
\text { pyrrolidine (toluene/tert-butanol, CuI, IPEA, RT, } 24 \mathrm{~h} \text { ) }\end{array}$ \\
\hline & {$[(\mathrm{S})-\mathrm{Py}]_{x}$-TPB-DMTP-COFs } & $\begin{array}{l}\text { Synthesis by click reaction of achiral }[\mathrm{HC} \equiv \mathrm{C}]_{x} \text {-TPBDMTP-COFs and }(S)-2-(\text { azido- } \\
\text { methyl)pyrrolidine (THF/water, CuI, } N, N \text {-diisopropylethylamine, RT, } 4 \mathrm{~h} \text { ) }\end{array}$ \\
\hline & $\mathrm{BIOMOL} \subset \mathrm{COF} 1$ & $\begin{array}{l}\text { Synthesized by dispersing NHS-functionalized COF-1 in lysozyme and peptide solution } \\
(0.1 \text { M MES buffer) }\end{array}$ \\
\hline & lipase PS@COFs & $\begin{array}{l}\text { Synthesis by shaking a mixture of achiral COFs and aqueous phosphate buffer solutions } \\
\text { of lipase PS }\left(500 \mathrm{rpm}, 25^{\circ} \mathrm{C}, 6 \mathrm{~h}\right)\end{array}$ \\
\hline & CD-COF-1,2 & $\begin{array}{l}\text { Synthesis by the thiol-ene click reactions between vinyl-functionalized COFs and } \\
\text { SH- } \beta \text {-CD (trifluorotoluene, AIBN, } 80^{\circ} \mathrm{C}, 48 \mathrm{~h} \text { ) }\end{array}$ \\
\hline \multirow[t]{3}{*}{$\begin{array}{l}\text { Direct synthesis } \\
\text { (achiral skeleton } \\
\text { monomers) }\end{array}$} & LZU-72-Boc & $\begin{array}{l}\text { LZU-72-boc and LZU-76-Boc are synthesized by the solvothermal method } \\
\text { (N-Boc-protected chiral monomers, triformylphloroglucinol, proper organic solutions, } \\
3 \mathrm{M} \text { aqueous acetic acid, } 90^{\circ} \mathrm{C}, 3 \text { days) }\end{array}$ \\
\hline & LZU-76-Boc & LZU-72 is obtained by deprotection of LZU-72-boc (heated at $245{ }^{\circ} \mathrm{C}, 15 \mathrm{~min}$ ) \\
\hline & LZU-72 & LZU-76 is obtained by deprotection of LZU-76-boc ( $4 \mathrm{M} \mathrm{HCl} / 1,4$-dioxane, RT, $2 \mathrm{~h}$ ). \\
\hline
\end{tabular}

Ref.
72
73
74
75
76
77

MH-CCOF1,2,3,4 Synthesized by the solvothermal method (chiral monomers derived from DBCBI, TAPB, TAH-CCOF1,2 ethanol/mesitylene, aqueous acetic acid, $120{ }^{\circ} \mathrm{C}, 3$ days)

SAH-CCOF1-Boc G-CCOF1 CTpPa-1

BtaMth COF

CCOF-MPC

CCOF-CuTPP

CCOFs 9-12

CCOFs 9-12/TPB

CCOFs $9^{\prime}-12^{\prime} / \mathrm{TPB}$ Tfp2-COF

Synthesized by the solvothermal method (chiral monomer CTp, Pa-1, ethanol/THF, $80^{\circ} \mathrm{C}$, $4 \mathrm{~h}$ )

Synthesized by the solvothermal method (1,3,5-benzenetricarboxaldehyde, chiral 79 monomer Mth, 1,4-dioxane/mesitylene, $3 \mathrm{M}$ acetic acid, $110{ }^{\circ} \mathrm{C}$, 3 days)

Synthesized by the solvothermal method (cyanuric chloride, $S$-(+)-2-methylpiperazine, 1,4-dioxane, $\mathrm{K}_{2} \mathrm{CO}_{3}, 90{ }^{\circ} \mathrm{C}, 36 \mathrm{~h}$ )

Synthesized by the solvothermal method $\left(S-(+)-2\right.$-methylpiperazine, $\mathrm{K}_{2} \mathrm{CO}_{3}, \mathrm{Pd}\left[\mathrm{P}(\mathrm{Ph})_{3}\right]_{4}$, TBrPP, 1,4-dioxane, $90{ }^{\circ} \mathrm{C}$ for 3 days and RT for 2 days)

CCOFs 9-12 are synthesized by the solvothermal method (TPBn, 2,5-

dimethoxyterephthalaldehyde (DMTA), proper solvents, acetic acid, $100{ }^{\circ} \mathrm{C}, 8 \mathrm{~h}$ )

CCOFs 9-12/TPB are synthesized by the solvothermal method (TPB1, TPBn, DMTA, proper solvents, acetic acid, $100{ }^{\circ} \mathrm{C}, 8 \mathrm{~h}-3$ days)

CCOFs $9^{\prime}-12^{\prime}$ /TPB are synthesized by the deprotection of CCOFS 9-12/TPB (base or acid)

Synthesized by the solvothermal method (chiral monomer Tfp2, 1,3,5-tris(4-

aminophenyl)benzene (TPB1), 1,4-dioxane/mesitylene, $3 \mathrm{M}$ acetic acid, $120{ }^{\circ} \mathrm{C}, 3$ days)

Synthesized by the solvothermal method $\left((R, R)\right.$-TTA or $(R, R)$-TTPA, $4,4^{\prime}-$

diaminodiphenylmethane, 1,4-dioxane, $3 \mathrm{M}$ acetic acid, $120{ }^{\circ} \mathrm{C}, 72 \mathrm{~h}$ )

Direct synthesis

CCOF-1,2

Synthesized by the solvothermal method (THB or TTHB, $\mathrm{Zn}(\mathrm{OAc})_{2} \cdot 2 \mathrm{H}_{2} \mathrm{O},(1 R, 2 R)-(-)-1,2-$ diaminocyclohexane, mesitylene/EtOH or DMF/EtOH, $6 \mathrm{M}$ acetic acid, $120{ }^{\circ} \mathrm{C}, 3$ days)

CCOF 3,4

CCOF 5 is synthesized by the solvothermal method $((R, R)$-TTA, tetra(4-anilyl)methane, 1,4-dioxane, $6 \mathrm{M}$ acetic acid, $120{ }^{\circ} \mathrm{C}, 3$ days)

CCOF 5,6

CCOF 6 is synthesized by the oxidation of CCOF 5 (2-methyl-2-butene, aqueous NaCl solution, glacial acetic acid, RT, $24 \mathrm{~h}$ )

CCOF 7,8

Synthesized by the solvothermal method ( $(R)$-BINOL-DA, TPE-TAM or iPr-TAM,

ethanol/mesitylene, $9 \mathrm{M}$ acetic acid, $120{ }^{\circ} \mathrm{C}$, 3 days)

$\beta$-CD COF

Fabricated by room temperature synthesis (Am7CD, TPA, ethanol, water, acetic acid, RT, 2 h)

CD-COFs

Chiral (A)- and (4)-CCOFs Synthesized by the microwave assisted method $\left(\gamma-\mathrm{CD}, \mathrm{B}(\mathrm{OMe})_{3}, \mathrm{LiOH}\right.$, mesitylene, DMF, $120{ }^{\circ} \mathrm{C}, 4 \mathrm{~h}$ with stirring)

Synthesized by the solvothermal method (Tp, triamine, and $(S)$ - or $(R)$-1-PEA, mesitylene/dioxane, $6 \mathrm{M}$ aqueous acetic acid, $120^{\circ} \mathrm{C}, 3$ days)

isotherms and pore size distribution profiles indicated a decrease in the surface area and pore diameter upon PSM. Because of the bulkiness of $\beta-\mathrm{CD}$ relative to the COF channel diameter, the loading of $\mathrm{CD}$ in the COF is limited to $\sim 3-6 \mathrm{wt} \%$. Even at such low loading, CD-COF-1 exhibits high enantioselectivity for chiral recognition.

The aforementioned case studies demonstrated the power of PSM in synthesizing CCOFs from achiral COF precursors. However, there are several limitations. First, there is no guarantee that chiral functional groups introduced in a COF are distributed in a homogeneous fashion. Second, upon introducing chiral auxiliaries post-synthetically, the material surface area, crystallinity, and chemical stability can be affected. In some cases, the resulting materials became amorphous. Third, the scope of chiral appendants is limited by their size and shape. These appendants need to be smaller than the pore-limiting diameter of the frameworks so that they can diffuse into COF pores. Some of these limitations can be addressed using direct synthesis discussed below.

\subsection{Direct synthesis}

Direct synthesis of CCOFs from enantiopure building blocks is a promising but challenging approach. Compared to PSM strategies, CCOFs obtained by direct synthesis show well-defined 

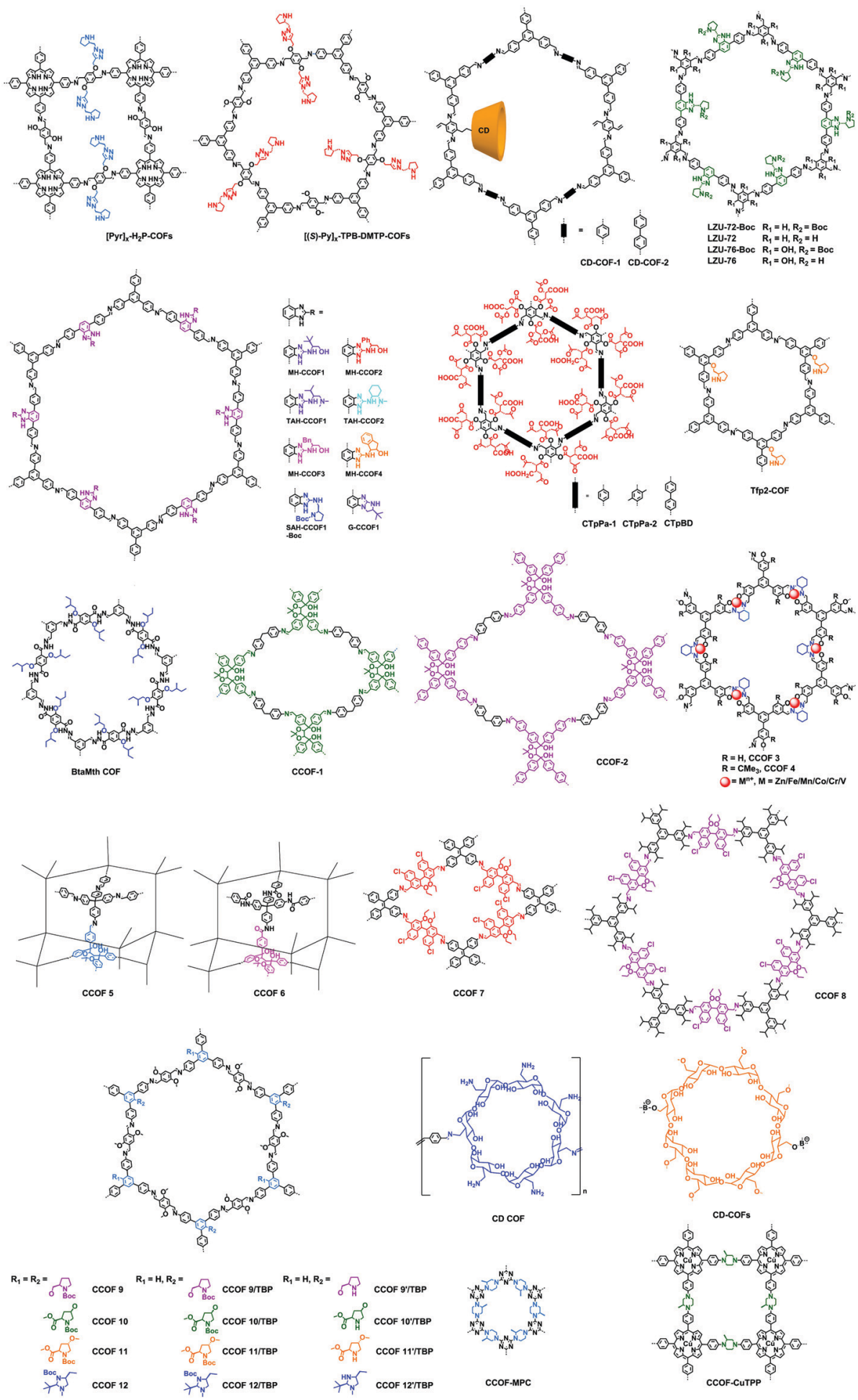

Fig. 2 Summary of CCOFs.

structures with precise and uniform distribution of chiral sites. Chiral COF monomers can be divided into two structural categories: (1) monomers with a chiral skeleton and (2) monomers with an achiral skeleton and chiral substituents.

CCOFs with an achiral skeleton are synthesized using monomers that are pre-modified with chiral functional groups.
Consequently, these CCOFs feature an achiral global structure with chiral functional groups appended in their cavities. CCOFs with a chiral skeleton are prepared by a direct assembly of the optically active organic monomers as building units. The resulting chiral skeletons engender CCOFs with unique chiral functions. 


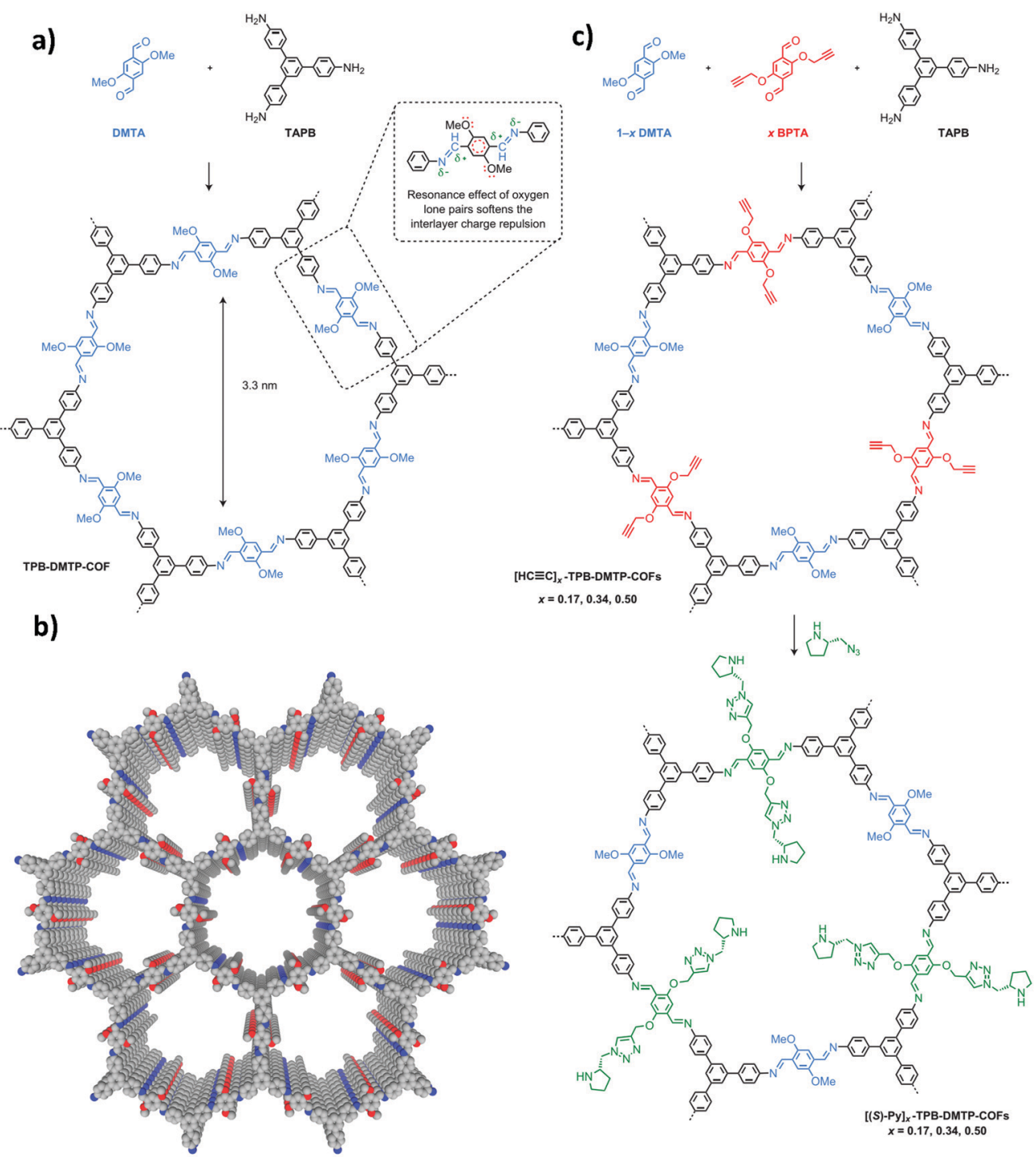

Fig. 3 PSM strategies for the synthesis of chiral COFs via channel-wall engineering by click reaction. Adapted from ref. 73 with permission from Nature Research, copyright 2015.

2.2.1 Direct synthesis from achiral skeleton monomers. Based on reticular chemistry principles, symmetric crystalline structures are most likely to form when using high-symmetry building units. ${ }^{106-108}$ One might expect that crystallization of chiral COFs from achiral organic monomers that are prefunctionalized with chiral functional groups can facilitate the crystallization in higher-symmetry space groups because these monomers often exhibit more rigid and symmetrical structures.

The first example of direct synthesis of chiral COFs was reported in 2016 by Wang and co-workers. ${ }^{85}$ They synthesized two chiral COFs, LZU-72 and LZU-76, directly from a chiral pyrrolidine-embedded building block. A rigid scaffold building block, $(S)$-4,4'-(1H-benzo[d]-imidazole-4,7-diyl)dianiline, was designed and synthesized with the chiral pyrrolidine moieties attached, and was used to assemble two CCOFs. LZU-72 and LZU-76 are crystallized in $P 3$ space group with a 2D layered hexagonal structure. Compared to the PSM method, this method could accurately control the distribution of chiral catalytic sites and maximize the number of chiral catalytic sites per unit cell. Subsequently, in 2019, the Wang group developed a general strategy to introduce various chiral functionalities in divergent COF monomers. As shown in Fig. 6, they constructed a series of CCOFs for asymmetric catalysis. ${ }^{86}$ Eight chiral monomers were synthesized from an achiral platform molecule DBCBI (4,7-dibromo-2-chloro-1 $H$-benzo[d]imidazole) by nucleophilic substitution and Suzuki coupling. The nucleophilic substitution introduces functionalities into the key platform molecule, and then Suzuki coupling creates a multi-connectivity of these DBCBI-based monomers. Then, eight CCOFs are constructed by dynamic covalent assembly of these chiral monomers and 1,3,5-tris(4-aminophenyl)-benzene (TAPB), and all crystallized in $P 3$ space group with a 2D layered hexagonal network. This threestep transformation (nucleophilic substitution, Suzuki coupling, 

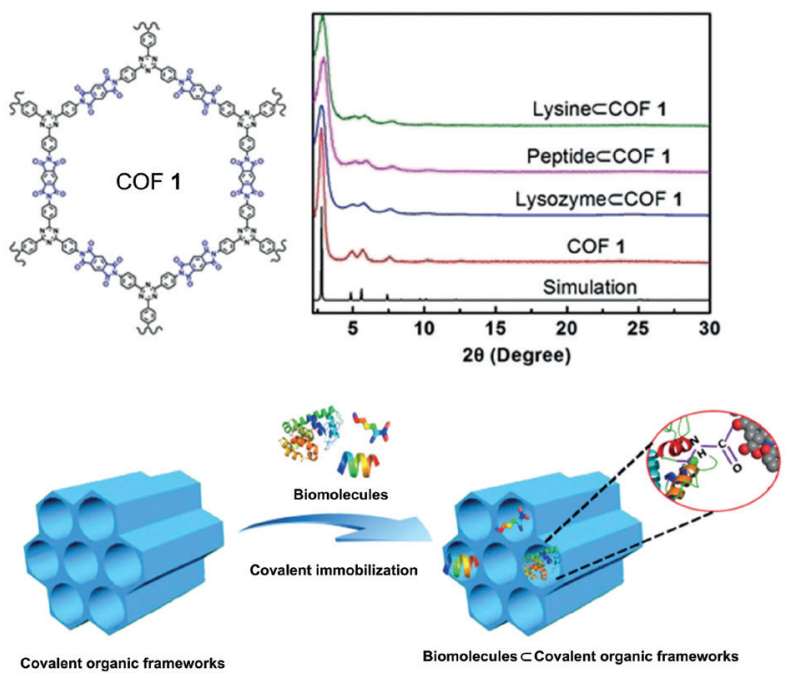

Fig. 4 Covalently immobilizing biomolecules in COFs. Reproduced from ref. 74 with permission from Wiley, copyright 2018.

and imine formation) from key platform molecules to CCOFs provides a general synthetic method for further high throughput screening and structure-activity investigation of COFs.

Another example of the bottom-up strategy that utilizes achiral skeleton monomers was reported by the Yan group in $2016 .{ }^{28}$ They first synthesized a chiral monomer CTp by functionalizing 1,3,5-triformylphloroglucinol (Tp) with chiral $(+)$-diacetyl-L-tartaric anhydride ((+)-Ac-L-Ta). Then, 2D hexagonal CCOFs (CTpPa-1, CTpPa-2 and CTpBD) were synthesized by condensing CTp with 1,4-phenylenediamine (Pa-1), 2,5-dimethyl$p$-phenylenediamine ( $\mathrm{Pa}-2)$ and benzidine (BD), respectively. Furthermore, they developed an in situ growth method to fabricate chiral COF-bound capillary columns for enantioselective gas chromatography.

Similar to Yan's work, the same synthetic strategy for CCOFs was implemented by the Zhang group in $2017 .^{79}$ They reported a hydrazone-linked CCOF in $\mathrm{PG}_{3}$ space group with a $2 \mathrm{D}$ layered hexagonal network from a chiral hydrazide building block with (S)-2-methylbutoxy groups attached. Moreover, they presented a one-pot synthetic method to fabricate the $\mathrm{CCOF} @ \mathrm{SiO}_{2}$ composite by adding $\mathrm{SiO}_{2}-\mathrm{NH}_{2}$ into the crystalline system for efficient high-performance liquid chromatography (HPLC) separation.

Cui, Liu, and co-workers reported a multivariate strategy to construct a series of two- and three-component 2D CCOFs in 2017 (Fig. 7). ${ }^{29}$ Based on $C_{3}$-symmetric 1,3,5-tris(4-aminophenyl) benzene as the backbone, L-proline and L-imidazolidine groups were attached on the central aromatic ring to afford chiral triamine scaffolds. A family of two- or three-component CCOFs with hexagonal channels was successfully prepared by crystallizing mixtures of triamines with and without chiral organocatalysts and with dimethoxyterephthalaldehyde (DMTA). As shown in Fig. 7, attempt to synthesize CCOFs $9^{\prime}-12^{\prime}$ via deprotection of two-component CCOFs 9-12 was unsuccessful. However, when tritopic monomers were doped with unfunctionalized analogue TPB, multivariate CCOFs 9-12/TPB were obtained. Subsequent deprotection reactions with TMSOTf or LiOH (aq) and TMSOTf delivered four catalytically active CCOFs $9^{\prime}-12^{\prime} /$ TPB. This multivariate strategy successfully circumvented the desymmetrizing effect caused by appending bulky chiral functional groups onto the monomers and consequently produced crystalline CCOFs that are otherwise difficult to form when functionalized monomers are used as the sole tritopic building block. Framework stability and crystallinity decreased monotonically upon increasing the loading of the chiral catalytic units.

Using the same strategy, the same group reported two other 2D CCOFs in $2019 .{ }^{81}$ These COFs are built from triamine or trialdehyde units that are pre-functionalised with organocatalysts DHIP and pyrrolidines. These catalytic units are anchored on the channel walls while the backbones of these COFs are achiral. These two CCOFs are shown to be efficient, metal-free, reusable and sustainable heterogeneous catalysts for asymmetric nucleophilic additions of aldehydes.

In 2017 and 2019, Dong, Chen, and co-workers reported two homochiral COFs by directly assembling the chiral organic component of $S$-(+)-2-methylpiperazine ( $S$-MP) and cyanuric chloride or copper tetrabromophenolphthalein (Cu-TBrPP). ${ }^{82,83}$ Cyanuric chloride and $\mathrm{Cu}-\mathrm{TBrPP}$ are linked via S-MP into a $2 \mathrm{D}$ layer extended on the crystallographic $a b$ plane to form CCOFMPC ( $\mathrm{PG}_{3}$ space group with a hexagonal cavity) and CCOF-CuTPP

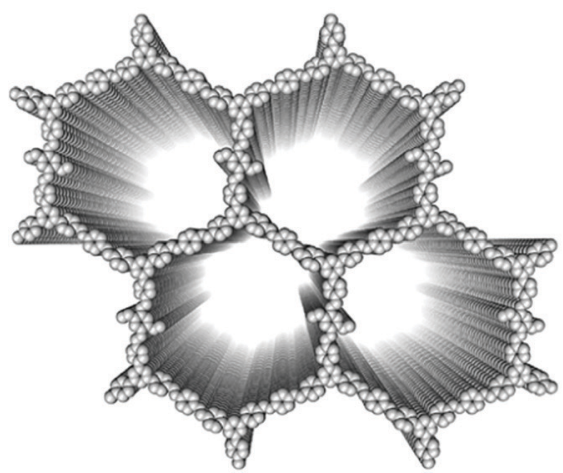

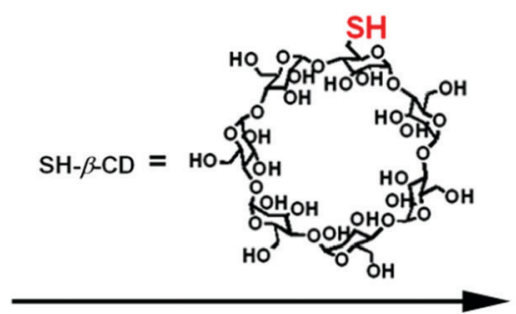

AIBN, $80^{\circ} \mathrm{C}$

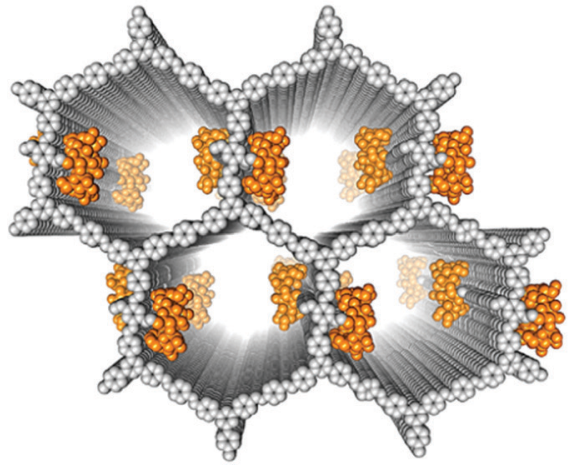

Fig. 5 Synthesis of achiral COFs and conversion to chiral CD-COFs via thiol-ene click reactions. Reproduced from ref. 76 with permission from the American Chemical Society, copyright 2019. 

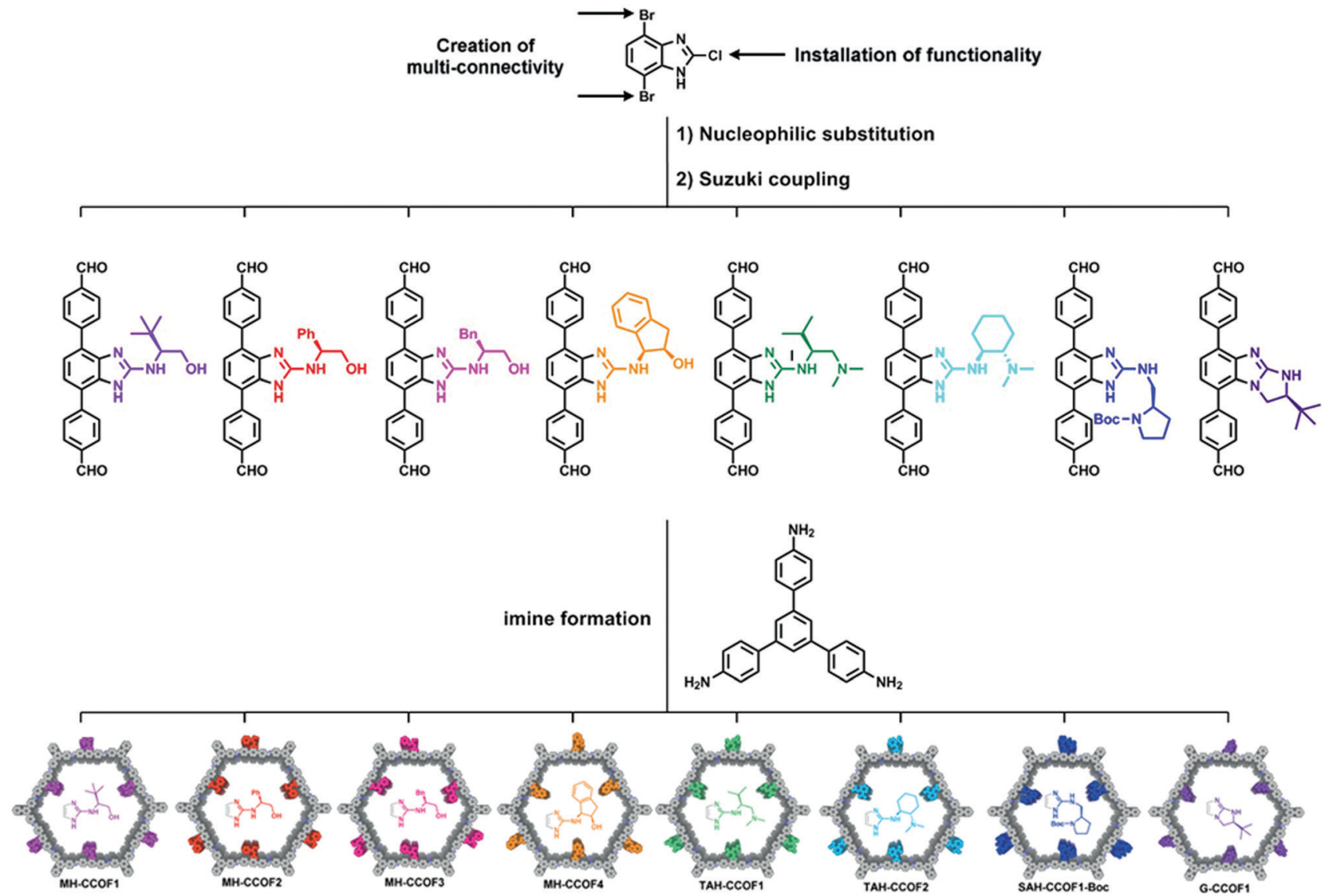

Fig. 6 Two-stage divergent strategy to construct multifarious CCOFs: (1) the divergent synthesis of chiral monomers by nucleophilic substitution and Suzuki coupling. (2) Synthesis of CCOFs by imine formation. Adapted from ref. 78 with permission from Wiley, copyright 2019.

$\left(P 4_{1}\right.$ space group with a tetragonal cavity), respectively. The chiralities of these CCOFs arise from the chiral centres of $S$-MP that are attached to the COF channel walls.

In fact, all the chiral COFs discussed above are prepared by using achiral skeleton monomers, with chiral functionalities. The chiral centres are attached or anchored on the surface of COF pores and channels, and the basic frameworks of CCOFs are still achiral, which could affect the chiral expression and limit the efficiency of chiral functions.

2.2.2 Direct synthesis from chiral skeleton monomers. It remains a stern challenge to synthesize CCOFs from chiral skeleton monomers due to the innate difficulty in controlling the crystallization of optically pure materials. In fact, before 2016, only a few CCOFs functionalized with (+)-diacetyl-Ltartaric anhydride and $(S)$-pyrrolidine were prepared by direct- or post-synthesis approaches. ${ }^{72,73}$ In both cases, the chiral functionalities are attached on achiral COF skeletons. It is ideal to synthesize CCOFs with chiral network structures by using chiral skeleton monomers. This strategy is expected to produce CCOFs with higher porosity, more open channels, and exhibiting chiral functions that are intrinsic to the chiral pore structures.

Towards this goal, in 2016, Cui et al. reported a series of CCOFs using monomers with chiral skeletons such as tetraaryl-1,3-dioxolane-4,5-dimethanols (TADDOL), metallosalen and 1,1'-bi-2-naphthol (BINOL) to construct CCOFs directly. ${ }^{84-87}$

TADDOL is a tartaric acid derivative with four aryl substituents in a propeller-type conformation. ${ }^{109-111}$ The 1,4-diol moiety in the TADDOL structure is helpful to form intramolecular hydrogen bonding and other intermolecular interactions, thereby providing structures with some rigidity. The Cui group reported the synthesis of two 2D imine-based CCOFs (CCOF-1 and CCOF-2) from two enantiopure TADDOL-derived tetraaldehydes (TTA and TTPA) and a flexible diamine linker (Fig. 8). ${ }^{84}$ In this work, because of the parallelogram arrangement of the coplanar aldehyde groups associated with a semirigid backbone and strong intermolecular interaction abilities, the enantiopure TADDOL-derivative became an intriguing CCOF building block. Powder X-ray diffraction and computer modeling together with pore size distribution analysis showed that both CCOF-1 and CCOF-2 crystallized in $P 2_{1}$ space group with a 2D layered tetragonal structure. Notably, CCOF-1 was doubly interpenetrated, whereas CCOF-2 was non-interpenetrated with an $\mathrm{AB}$ stacking mode.

The $N, N^{\prime}$-bis(salicylidene)ethylenediamine unit (salen) represents one of the most important ligands in coordination chemistry. ${ }^{112-117}$ Salen has the ability to stabilize metal ions in various oxidation states, which makes it an efficient catalyst for 


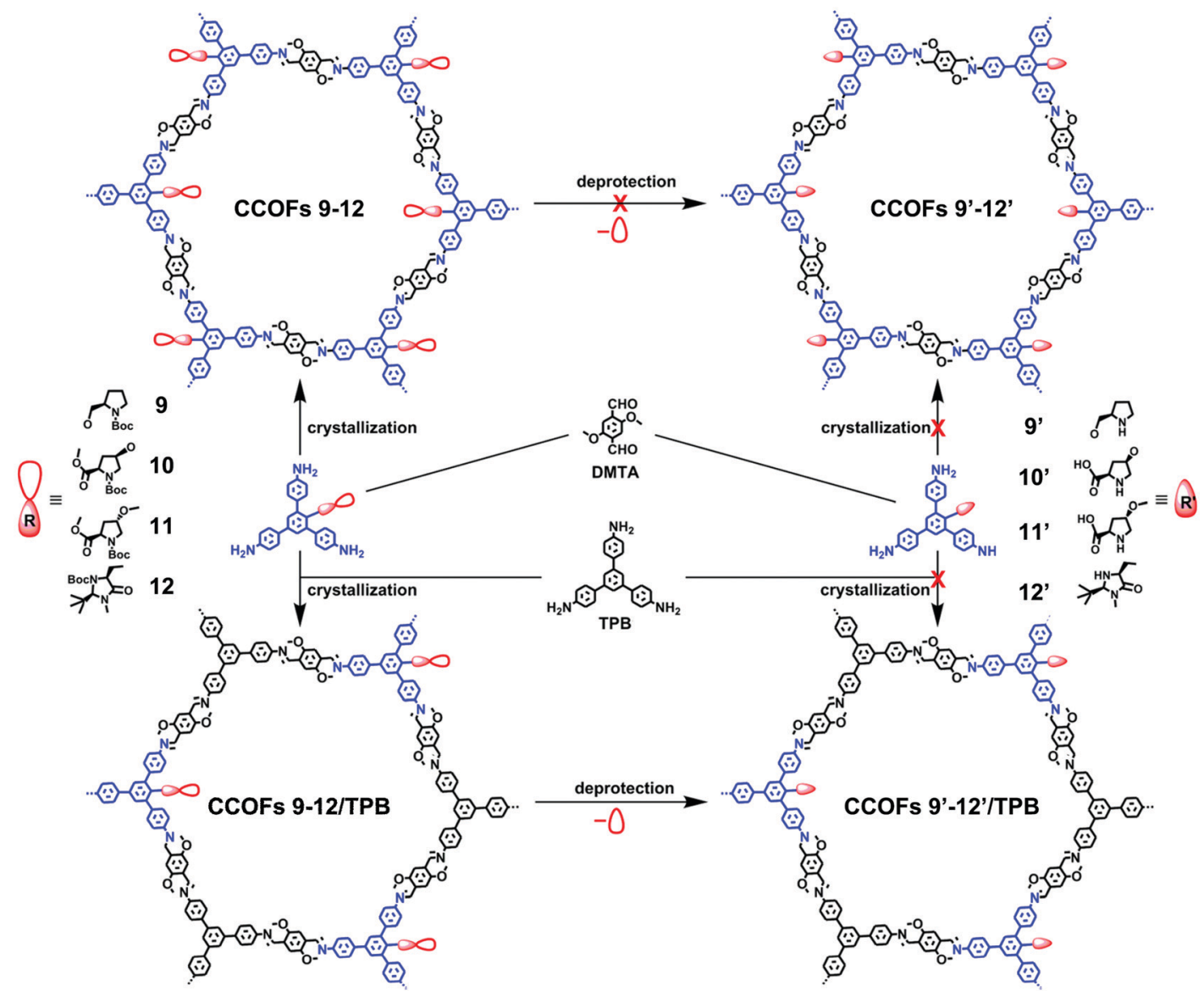

Fig. 7 Synthesis of CCOFs 9-12, CCOFs 9-12/TPB and CCOFs $9^{\prime}-12^{\prime} /$ TPB. Adapted from ref. 80 with permission from the American Chemical Society, copyright 2017.

numerous organic transformations. It is well-known that chiral salen ligands are privileged ligands for asymmetric catalysis. ${ }^{118-120}$ Salen was first introduced to the construction of COFs by the Wang group in 2017. ${ }^{121}$ They reported a Salenbased COF with high crystallinity and excellent stability via solvothermolysis. In addition, they metallated salen-COF with different metal ions for further applications. Nonetheless, the synthesis of CCOFs from $\mathrm{M}$ (salen) complexes has not been explored. Another example of a salen-based COF was reported by Yang and co-workers in $2019 .{ }^{122}$ Here, they developed an in situ salen formation method during the COF synthesis. In both cases, achiral salen-based monomers were used, and the resulting COFs showed high activity and stability as heterogeneous catalysts. To synthesize chiral COF analogues, in 2017, Cui, Liu, and co-workers turned their attention to chiral Salenbased monomers, and reported a new metal-directed synthesis method. Using this method, two 2D Zn(salen)-based CCOFs, CCOF 3 and CCOF 4, were synthesized. ${ }^{85}$ These CCOFs were prepared by imine formation of enantiopure 1,2-diaminocyclohexane with $C_{3}$-symmetric trisalicylaldehydes with or without tert-butyl groups (Fig. 9). Both CCOFs 3 and 4 crystallized in $P 321$ space group with a 2D layered hexagonal network. This is the first report of utilizing chiral salen as a linkage to form CCOF structures. All of them can even be regarded as crystalline materials constructed from chiral salen moieties being connected via $\mathrm{C}-\mathrm{C}$ bonds as well. When comparing CCOF 4 with CCOF 3, the bulky hydrophobic groups in the COF structure are capable of protecting hydrolytically susceptible backbones via kinetic blocking, and thus, the stability of CCOF 4 in strong acid and alkaline conditions was drastically improved.

Notably, the $\mathrm{Zn}$ (salen) modules in the CCOFs provide a site to introduce multivariate metals into the frameworks by postsynthetic metal exchange. The CCOFs after metal exchange maintain high crystallinity and porosity and can serve as stable and efficient metallocatalysts.

Enantiopure BINOL is one of the most important sources of chirality for organic and materials chemistry. ${ }^{123-126}$ However, it was not used to construct CCOFs until Cui and co-workers reported two chiral BINOL-based CCOFs (CCOF 7 and CCOF 8) by condensing an elaborately designed, optically pure BINOL-based linear dialdehyde with tetrakis(4-aminophenyl)ethane or 1,3,5tris(3,5-diisopropyl-4-aminophenyl)benzene (Fig. 10). ${ }^{87}$ CCOF 7, modelled in $C 2$ space group, exhibits $2 \mathrm{D}$ layered tetragonal networks that are stacked in a slipped AA fashion. Conversely, CCOF 8 in $R 3$ space group shows a staggered stacking (ABC) mode of $2 \mathrm{D}$ hexagonal layered networks. Importantly, the CCOF constructed from BINOL-based dialdehyde and tetraphenylethylene units can be readily exfoliated into ultrathin $2 \mathrm{D}$ CCOF nanosheet materials. 


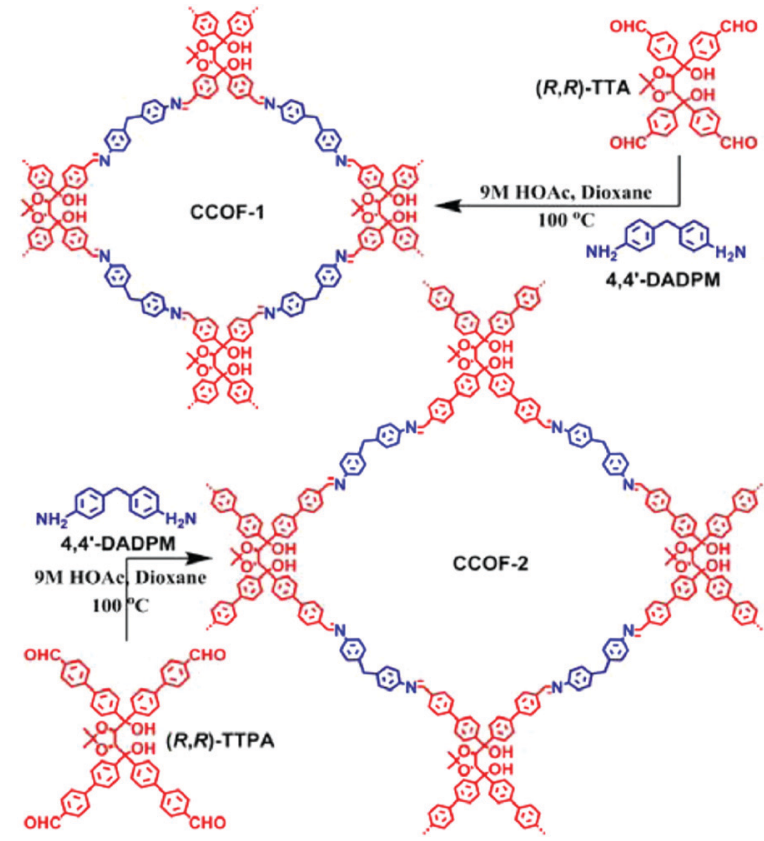

Fig. 8 Synthesis of CCOF-1 and CCOF-2. Adapted from ref. 84 with permission from the American Chemical Society, copyright 2016.

$\beta$-Cyclodextrin ( $\beta$-CD) is one of the most employed supramolecules which bears a hydrophobic cavity with a fascinating intrinsically chiral cavity. ${ }^{103-105}$ In 2018, the Feng group reported the synthesis of a $\beta$-CD COF by using heptakis(6-amino-6-deoxy)- $\beta$-CD and terephthalaldehyde through acetic acid catalyzed polycondensation in water and ethanol at room temperature. ${ }^{89}$ Because of the direct synthesis based on the asymmetric $\beta$-CD building blocks, a great amount of $\mathrm{CD}$ units can be introduced into the material. The $\beta$-CD COF exhibited high thermal stability, uniform pore size distribution, and a large surface area.

Compared to 2D COFs, 3D COFs have more complex structures with diverse topologies. They typically exhibit larger surface areas, higher accessible sites per volume and fascinating confinement effects. These features promise 3D COFs in multifarious applications. ${ }^{127-129}$ In 2018, a 3D homochiral COF (CCOF 5) was synthesized by imine condensation of a TADDOL-derived tetraaldehyde (TTA) with a $T_{\mathrm{d}}$-symmetrical tetrahedral monomer, tetra(4-anilyl)methane (TAM) (Fig. 11). ${ }^{86}$ Moreover, CCOF 5 could transform into an amide-linked 3D CCOF (CCOF 6) via postsynthetic oxidation. Such treatment enhanced chemical stability without affecting its crystallinity and porosity. Crystalline structures of the CCOFs were determined by powder X-ray diffraction (PXRD) and verified by pore size distribution analysis. The isostructural COF 5 and CCOF 6 feature a 4-fold interpenetrated diamondoid open framework with tubular channels decorated with chiral dihydroxy auxiliaries. This work also demonstrates the power of PSM in CCOF to CCOF conversion while maintaining chirality, structural integrity, crystallinity, and porosity.

In 2017, Wang et al. reported the construction of a polymeric and periodic 3D CCOF by using the reaction between a flexible and aliphatic macrocycle, namely $\gamma$-cyclodextrin $(\gamma-\mathrm{CD})$, and trimethyl borate $\left(\mathrm{B}(\mathrm{OMe})_{3}\right){ }^{90}$ This $\mathrm{CD}-\mathrm{COF}$ has an anionic tetrakis(spiroborate) linkage with a tetrahedral structure (Fig. 12).

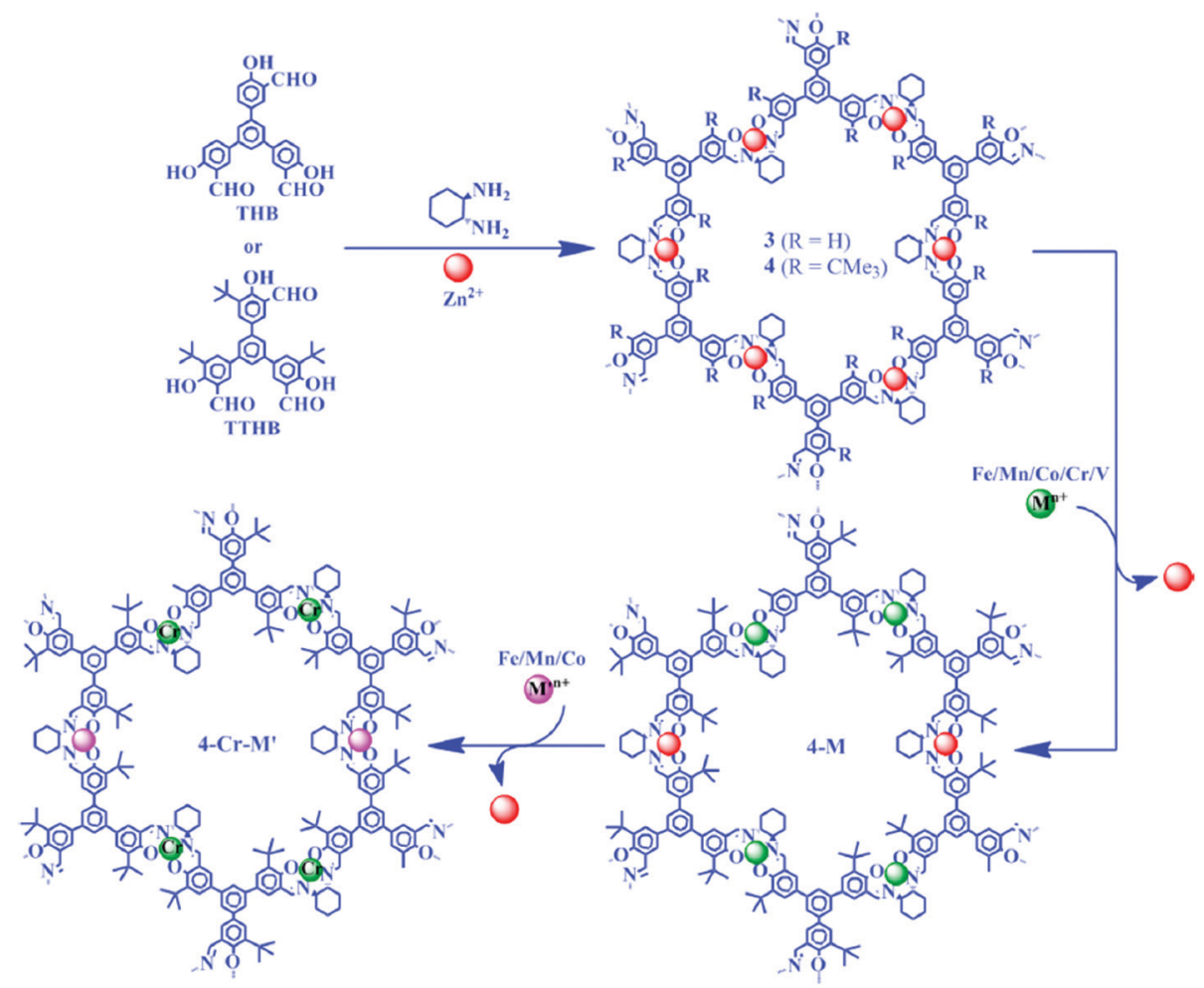

Fig. 9 Synthesis of CCOF 3 and CCOF 4. Adapted from ref. 85 with permission from the American Chemical Society, copyright 2017. 

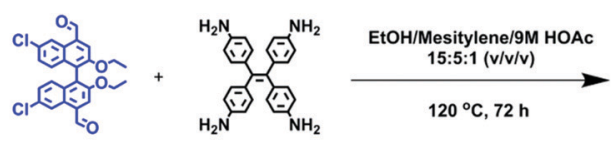

BINOL-DA

$120^{\circ} \mathrm{C}, 72 \mathrm{~h}$

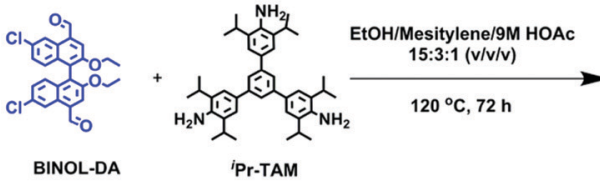

Fig. 10 Synthesis of CCOF-7 and CCOF-8. Reproduced from ref. 87 with permission from the American Chemical Society, copyright 2019

a)

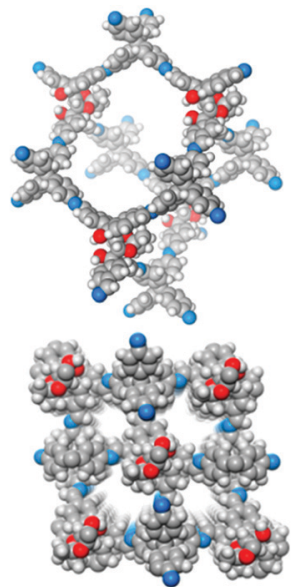

b)

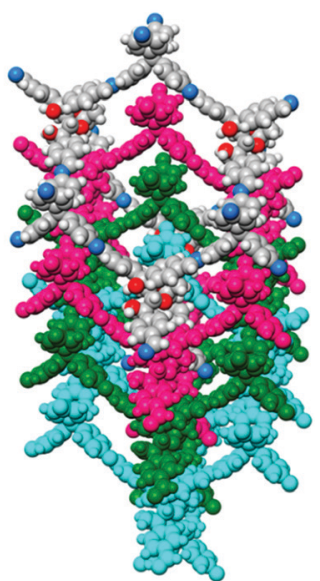

Fig. 11 Structural representation of CCOF 5. (a) Adamantine-like cage in the diamond net. (b) Structure viewed along the a-axis showing 4-fold interpenetration of a diamond net. (c) Two kinds of 1D channels long the $\mathrm{C}$-axis. $\mathrm{C}$ gray; $\mathrm{N}$ blue; $\mathrm{H}$ white; $\mathrm{O}$ red. Adapted from ref. 86 with permission from the American Chemical Society, copyright 2018.

The CD-COF requires counter ions to balance its negative charges and thus offers opportunities to adjust the inner surface environment by selecting the counter ions in the charged pore surface.

\subsection{Chiral induction synthesis}

Theoretically, chirality occurs when a compound has only rotational and translational symmetry and no roto-inversions. ${ }^{130-132}$ When discussing chirality in solid materials, there are two different aspects that need to be considered: first, whether
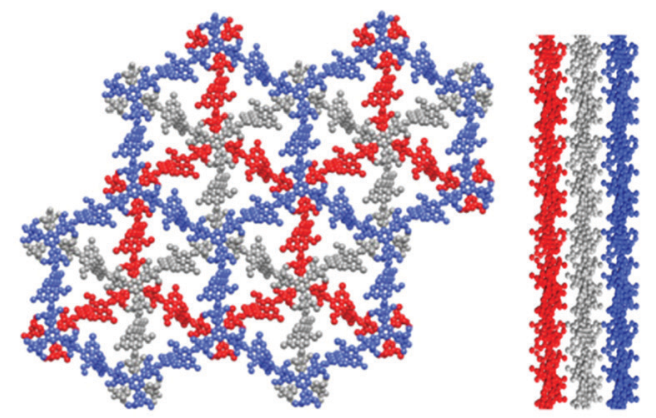

CCOF 8
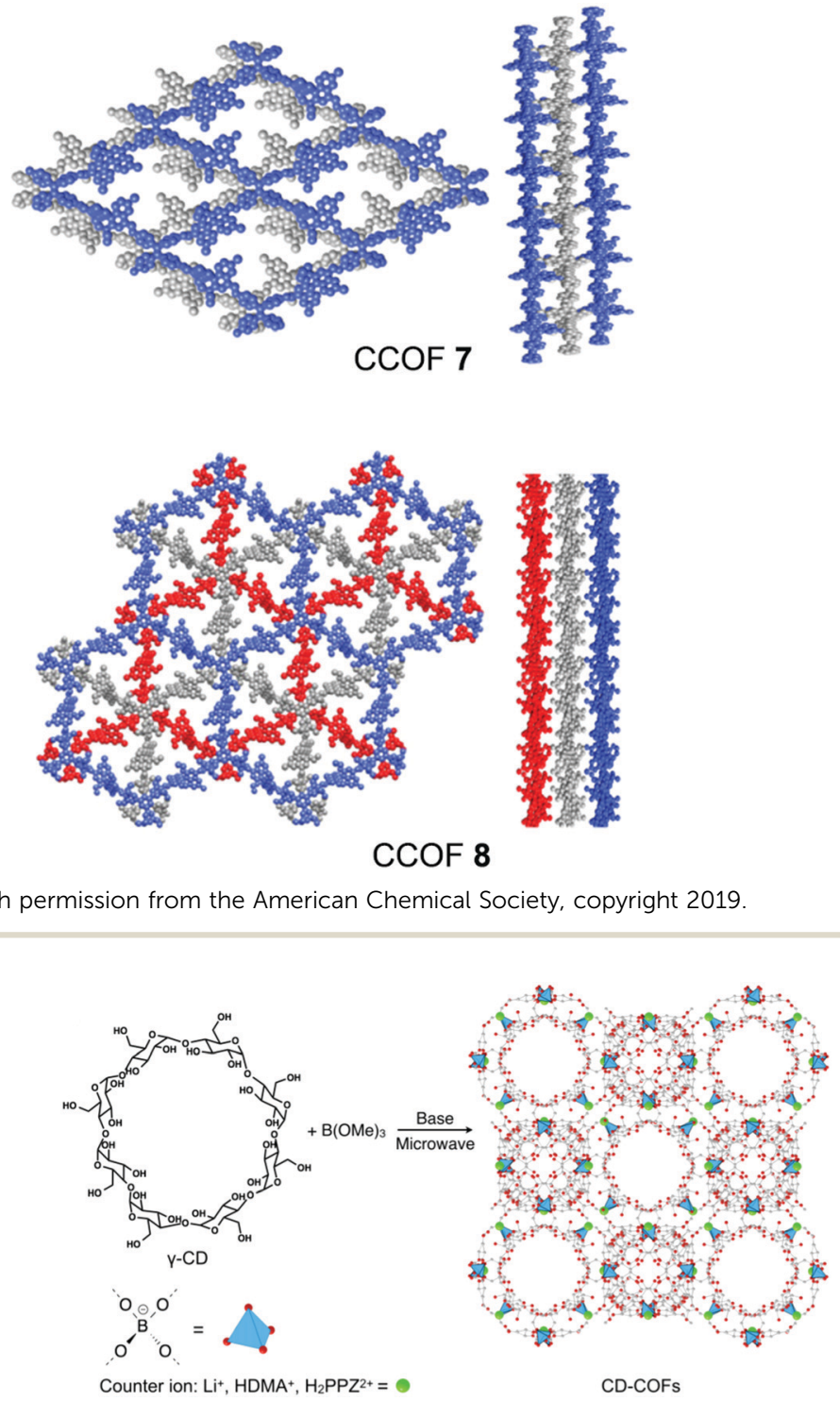

Fig. 12 Synthesis of CD-CCOFs. Adapted from ref. 90 with permission from Wiley, copyright 2017

the components (building units) of the structure are asymmetrical themselves; second, whether the arrangement of components into the materials is chiral. ${ }^{14}$ Thus, there is nothing intrinsic regarding COFs that precludes their structures from being chiral, even when building units themselves are achiral. Many COF materials have asymmetric structures in their frameworks, but always form only as a racemic conglomerate containing both the left- and right-handed sections in bulk solids at the ratio of $1: 1{ }^{133}$ Chiral induction, however, can potentially bias this equal probability process to increase the population of one-handed structure over the other. There are already a few excellent examples of using the phenomenon of chiral induction to synthesize homochiral metal-organic materials and inorganic crystals such as $\mathrm{NaClO}_{3} \cdot{ }^{14,134-136}$

Inspired by the previous chiral induction works, homochiral crystallization of COFs from achiral precursors was realized by 
chiral catalysis in $2018 .^{88}$ The structure and chemical properties of chiral catalysts can be transcribed into COF pore shapes and properties. The advantage of chiral induction synthesis is that in principle it is not limited to any particular composition or structure type. Cui, Liu and co-workers reported a total of nine 2D CCOFs with controlled handedness which were solvothermally synthesized by imine condensation of $C_{3}$-symmetric 1,3,5-triformylphloroglucinol (Tp) with achiral diamine or triamine linkers in the presence of $(R)$ - or $(S)-1$ phenylethylamine (PEA) as a catalyst (Fig. 13). The enantiomer of 1-PEA was replaced by achiral monomers from the precursors, and the chiral memory left in the original position, thereby leading to a torsion structure. After that, the amorphous polymers crystallized and chiral COFs in $P 3$ or $P 6$ space group with a 2D layered hexagonal network formed.

Compared with other methods, chiral induction synthesis of CCOFs solely from achiral monomers is a very new direction and its potential has just being demonstrated. However, the scope of this method remains elusive due to the unpredictability of a suitable chiral catalyst for a given set of achiral building blocks. Thus, further studies on chiral induced crystallization of more CCOFs, especially 3D CCOFs, from achiral precursors and a detailed understanding of the enantioselective processes are greatly needed. Notably, in 2018, Wang, Yaghi and co-workers solved the single-crystal structure of a new CCOF LZU-111 with the rare lon-b-c3 topology. This chiral COF was also synthesized from achiral monomers. ${ }^{68}$ The origin of chirality was from threefold interpenetration; the intersections of the networks generate a $\sigma_{5}$ screw axis, and the formation of chiral LZU-111 was based on a self-enantioseparation process. This work could also elucidate the potential and challenges to control the self-enantioseparation process by the chiral induction method in the future.

\section{Applications}

\subsection{Asymmetric catalysis}

Functional porous materials such as MOFs, ${ }^{137-140}$ inorganic zeolites $^{141,142}$ and functional cages ${ }^{15,143-145}$ used for heterogeneous catalysis have been developed for decades. As emerging porous materials, COFs exhibit great potential to be functionalized as effective and robust heterogeneous catalysts for organo-, electro-, or photocatalytic applications. ${ }^{128,146-148}$ In 2011, Wang et al. for the first time demonstrated the utility of COFs as heterogeneous catalysts, which show high activity in catalyzing the Suzuki-Miyaura coupling reaction. ${ }^{149}$ Following this seminal work on the design and synthesis of catalytically active COFs, in 2014, Yan et al. synthesized 3D COFs for base-catalyzed Knoevenagel condensation reactions with high conversion. ${ }^{129}$ In the same year, Lotsch et al. reported a hydrazone-based COF for photocatalytic hydrogen production from water for the first time. ${ }^{150}$ In 2018, Yaghi et al. developed a 2D porphyrin-based COF for electrocatalytic reduction of $\mathrm{CO}_{2}$ to $\mathrm{CO}$ with high selectivity

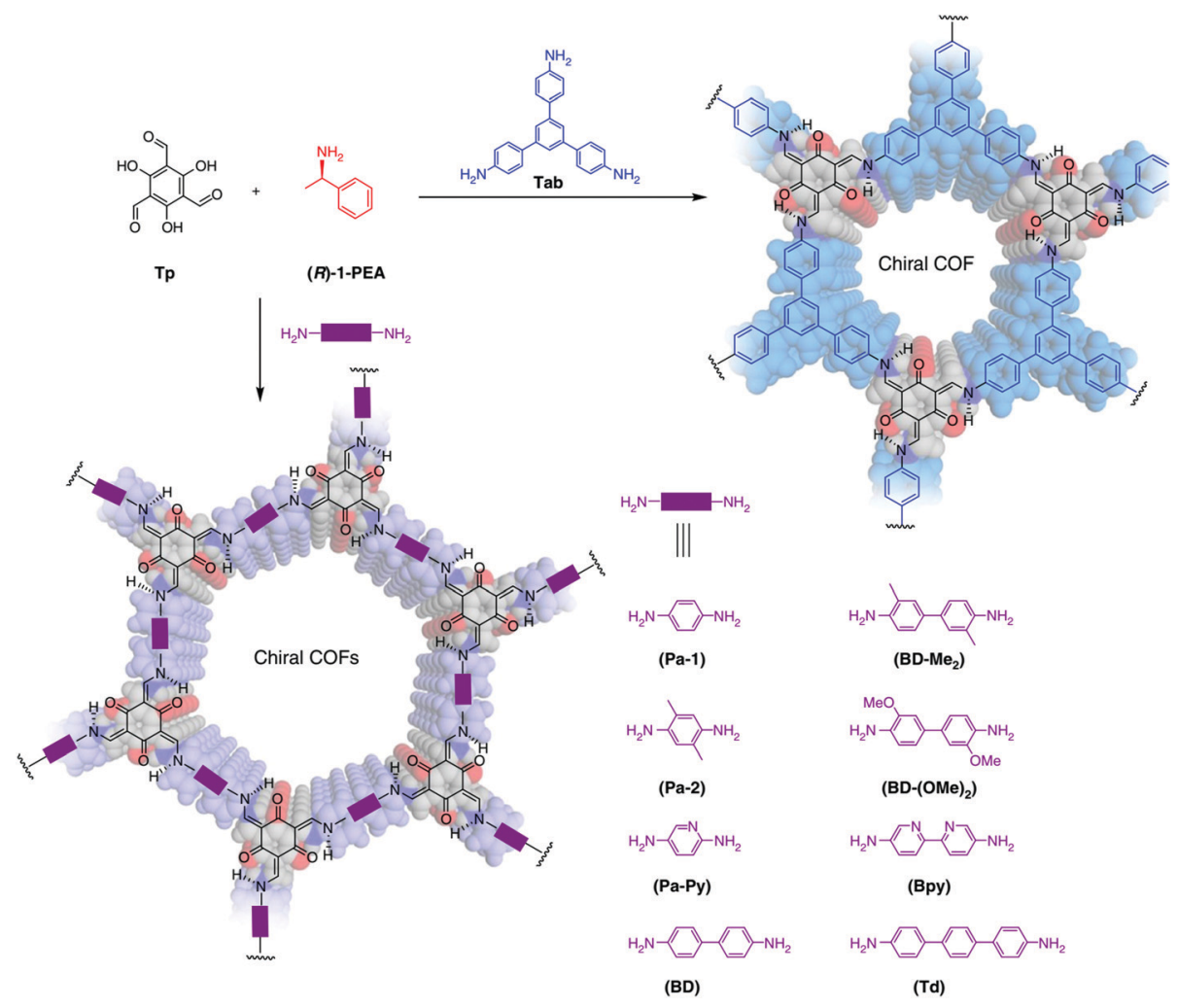

Fig. 13 Chiral induction synthesis of CCOFs in the presence of phenylethylamine (PEA). Adapted from ref. 88 with permission from Nature Research, copyright 2018. 
and efficiency. This work represents a study of direct electronic structure-function relationships of COF electrocatalysts. ${ }^{151}$

Chiral COFs have been demonstrated as effective heterogeneous catalysts for asymmetric reactions. Generally, CCOFs combine the advantages of both heterogeneous catalysts and homogeneous molecular catalysts. Specifically, they can be easily separated from the reaction mixture by filtration, and the collected solid catalysts are reusable. Moreover, the porous hosts offer isolation of individual catalytic units. When materials are highly porous, the diffusion rates of the substrates and the products are likely to be faster than the catalytic rate. Consequently, the catalytic activity might be comparable with homogeneous analogues. These features render CCOFs as valuable heterogeneous asymmetric catalysts. Nevertheless, research on chiral COFs for asymmetric catalysis is still in its infancy.

The earliest application of chiral COFs in asymmetric catalysis was reported by Jiang et al. in 2014. ${ }^{71}$ By controlled integration of chiral pyrrolidine sites into the pore walls of achiral imine-linked porphyrin COFs, CCOFs $\left([\mathrm{Pyr}]_{x}-\mathrm{H}_{2} \mathrm{P}-\mathrm{COF}\right.$, $X=25,50,75$, and $\left.100\left(X=0: \mathrm{H}_{2} \mathrm{P}-\mathrm{COF}\right)\right)$ were synthesized which were then applied as metal-free heterogeneous catalysts for asymmetric Michael addition reactions. These catalysts exhibited enantioselectivity (ee) and diastereoselectivity (dr) values of $49 \%$ and $70 / 30$. The optimized $[\mathrm{Pyr}]_{0.25}-\mathrm{H}_{2} \mathrm{P}-\mathrm{COF}$ was then used for the flow reaction system and the column maintained $100 \%$ conversion and stereoselectivity (44\% ee, 65/35 dr) for more than $48 \mathrm{~h}$ (Fig. 14). In 2015, the same research group reported a $2 \mathrm{D}$ imine-based COF, named TPB-DMTP-COF (TPB, triphenylbenzene; DMTP, dimethoxyterephthaldehyde), with high stability by incorporating methoxy groups into its pore walls to reinforce interlayer interactions. CCOFs $\left([(S)-\mathrm{Py}]_{x}-\mathrm{TPB}-\right.$ DMTP-COFs, $x$ is the percentage of functional groups, $x=0.17$, $0.34,0.50$ ) were synthesized via post-synthetic channel-wall engineering using a three-component condensation to obtain the intermediate $[\mathrm{HC} \equiv \mathrm{C}]_{x}$-TPB-DMTP-COFs. The following quantitative azide-ethynyl click reaction appended chiral unit $(S)$-pyrrolidine on their pore walls at various loadings. $[(S)-\mathrm{Py}]_{x^{-}}$ TPB-DMTP-COF retained its crystalline structure and was shown to be stable towards water, strong acids and strong bases. $[(S) \text {-Py }]_{x}$-TPB-DMTP-COFs were then used as asymmetric organocatalysts for Michael addition. These CCOF catalysts allowed Michael additions to be operated in neat water.

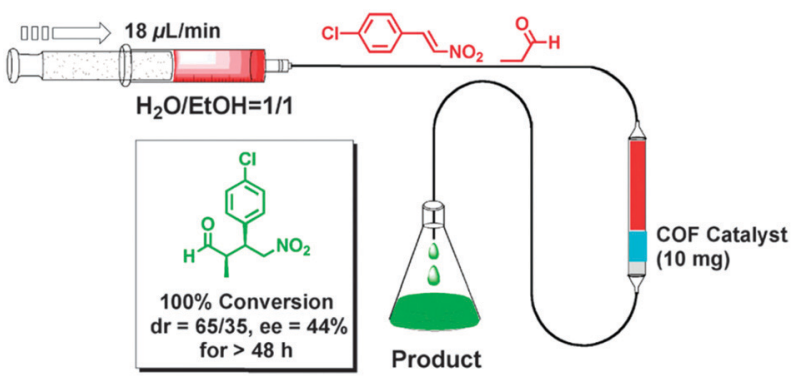

Fig. 14 The flow reaction system based on the $[\mathrm{Pyr}]_{25}-\mathrm{H}_{2} \mathrm{P}-\mathrm{COF}$ column for Michael addition reaction. Adapted from ref. 72 with permission from the Royal Society of Chemistry, copyright 2014.
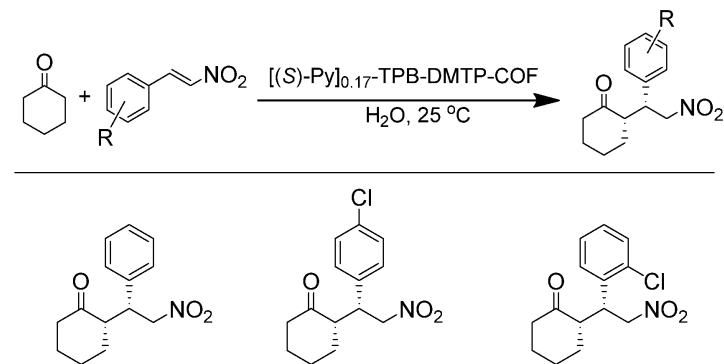

12 h, d.r. $=90 / 10$, ee $=92 \%$

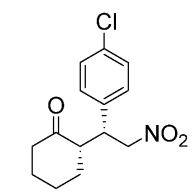

10 h, d.r. $=90 / 10$ ee $=90 \%$

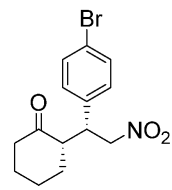

12 h, d.r. $=93 / 7$ ee $=95 \%$

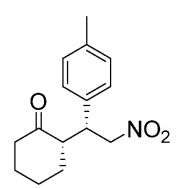

16 h, d.r. $=92 / 8$, $\mathrm{ee}=93 \%$

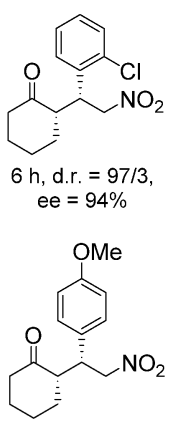

26 h, d.r. $=94 / 6$ $\mathrm{ee}=96 \%$

Fig. 15 Michael reactions catalyzed by $[(S)-P y]_{0.17}-$ TPB-DMTP-COFs.

This promoted the addition of cyclohexanone- and $\beta$-nitrostyrene-based compounds with $100 \%$ conversion and with an ee and $d r$ values of $90-96 \%$ and 90/10-97/3, respectively (Fig. 15). Besides, as a heterogeneous catalyst, $[(S) \text {-Py }]_{0.17}$-TPBDMTP-COF exhibits high recyclability, activity, enantioselectivity and diastereoselectivity after five cycles. Specifically, $[(S) \text {-Py }]_{0.34}$-TPB-DMTP-COF and $[(S) \text {-Py }]_{0.50}$-TPB-DMTP-COF are less active than $[(S) \text {-Py }]_{0.17}$-TPB-DMTP-COF. It took 17, 34, and 12 hours respectively for these catalysts to reach $100 \%$ conversion under the same reaction condition. This observation indicates that excessive catalytic sites on the channel walls after post-modification may block the pores and channels of CCOFs, thereby reducing the diffusion rates of the substrates (Fig. 16). ${ }^{73}$

In 2016, Wang et al. utilized the deprotected LZU-76, which was synthesized from the direct condensation of chiral pyrrolidine-embedded building block $(S)$-4,4'-(2-(pyrrolidin-2yl)-1H-benzo[ $d$ ]imidazole-4,7-diyl)dianiline with triformylphloroglucinol LZU-76, as a heterogeneous chiral catalyst to catalyze the asymmetric aldol reaction. The chiral-pyrrolidine catalytic site is evenly distributed on the inner pore wall of two CCOFs, thus providing convenient transportation for reactants and products. As shown in Fig. 17, the reaction produced aldol products with high enantioselectivity (up to $87 \%$ ee). Besides, LZU-76 could be reused at least 3 times without loss of enantioselectivity. ${ }^{85}$

Then, in 2019, this team developed a general strategy to introduce chirality in a divergent manner into COFs. Hydrogenbond-donor, Brønsted-acidic, and Brønsted-basic sites were then integrated into different CCOFs for heterogeneous asymmetric catalysis. TAH-CCOF1 and TAH-CCOF2 possess tertiaryamine and hydrogen-bonding sites and display high activity and enantioselectivity in the asymmetric amination reaction of ethyl 2-oxocyclopentane-1-carboxylate with di-tert-butylazodicarboxylate (up to $96 \%$ yield and $99 \%$ ee). The substrate generality was further confirmed by the reactions of other $\beta$-keto esters with 

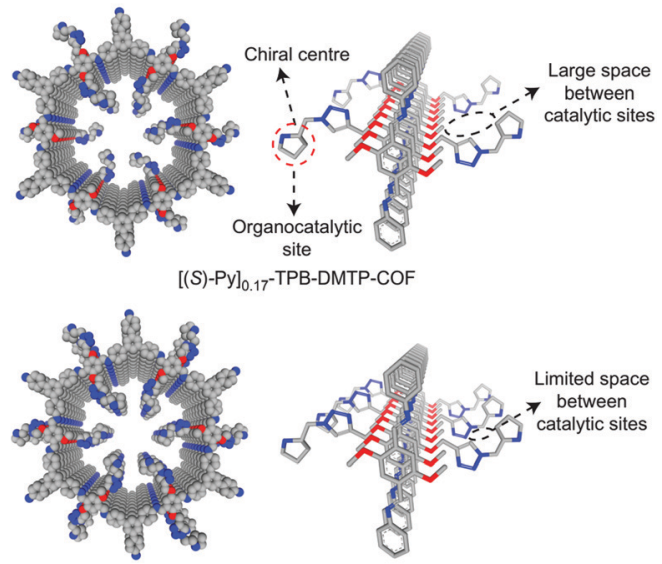

$[(S)-P y]_{0.34}$-TPB-DMTP-COF

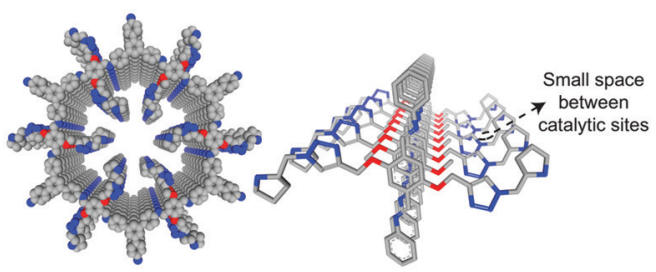

$[(S)-P y]_{0.50}$-TPB-DMTP-COF

Fig. 16 Channel-wall structure of $[(S)-P y]_{x}$-TPB-DMTP-COFs $(x=0.17$, 0.34 and 0.50 ). Adapted from ref. 73 with permission from Nature Research, copyright 2017.
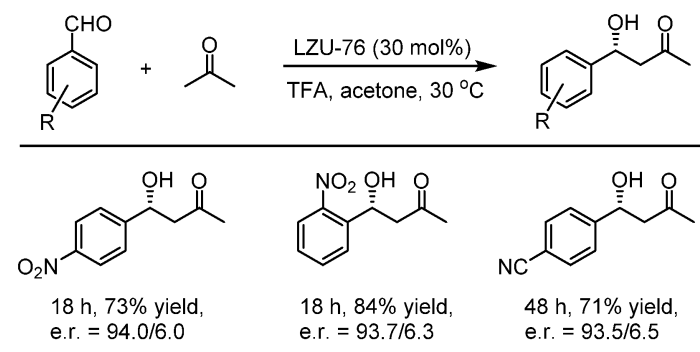

$18 \mathrm{~h}, 84 \%$ yield, e.r. $=93.7 / 6.3$

$48 \mathrm{~h}, 71 \%$ yield
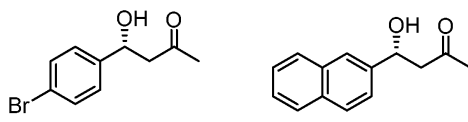

$96 \mathrm{~h}, 46 \%$ yield,

e.r. $=88.4 / 11.6$

$80 \mathrm{~h}, 25 \%$ yield,

e.r. $=93.1 / 6.9$

Fig. 17 Asymmetric aldol reaction catalyzed by LZU-76.

di-tert-butylazodicarboxylate, and up to $98 \%$ yield and $91 \%$ ee were obtained (Table 2). Additionally, the TAH-CCOF2 catalyst could be reused at least 7 times without obvious loss of enantioselectivity. ${ }^{86}$

After treatment with $\mathrm{Ti}\left(\mathrm{O}^{\mathrm{i}} \mathrm{Pr}\right)_{4}$, both CCOF-1 and $\mathbf{- 2}$ could catalyze the $\mathrm{Et}_{2} \mathrm{Zn}$ addition reactions of the substrates of aromatic aldehydes bearing electron-donating and electronwithdrawing groups with high conversion (96-99\%) and enantioselectivity (74-94\% ee) (Fig. 18), Furthermore, the catalysts were proved to be recyclable which could be recovered and reused for 4 runs without loss of activity. Besides, a sterically more demanding substrate of coronenyl aldehyde was used in the addition reaction and less than 5\% conversion
Table 2 Asymmetric amination of $\beta$-ketoesters catalyzed by CCOF catalysts

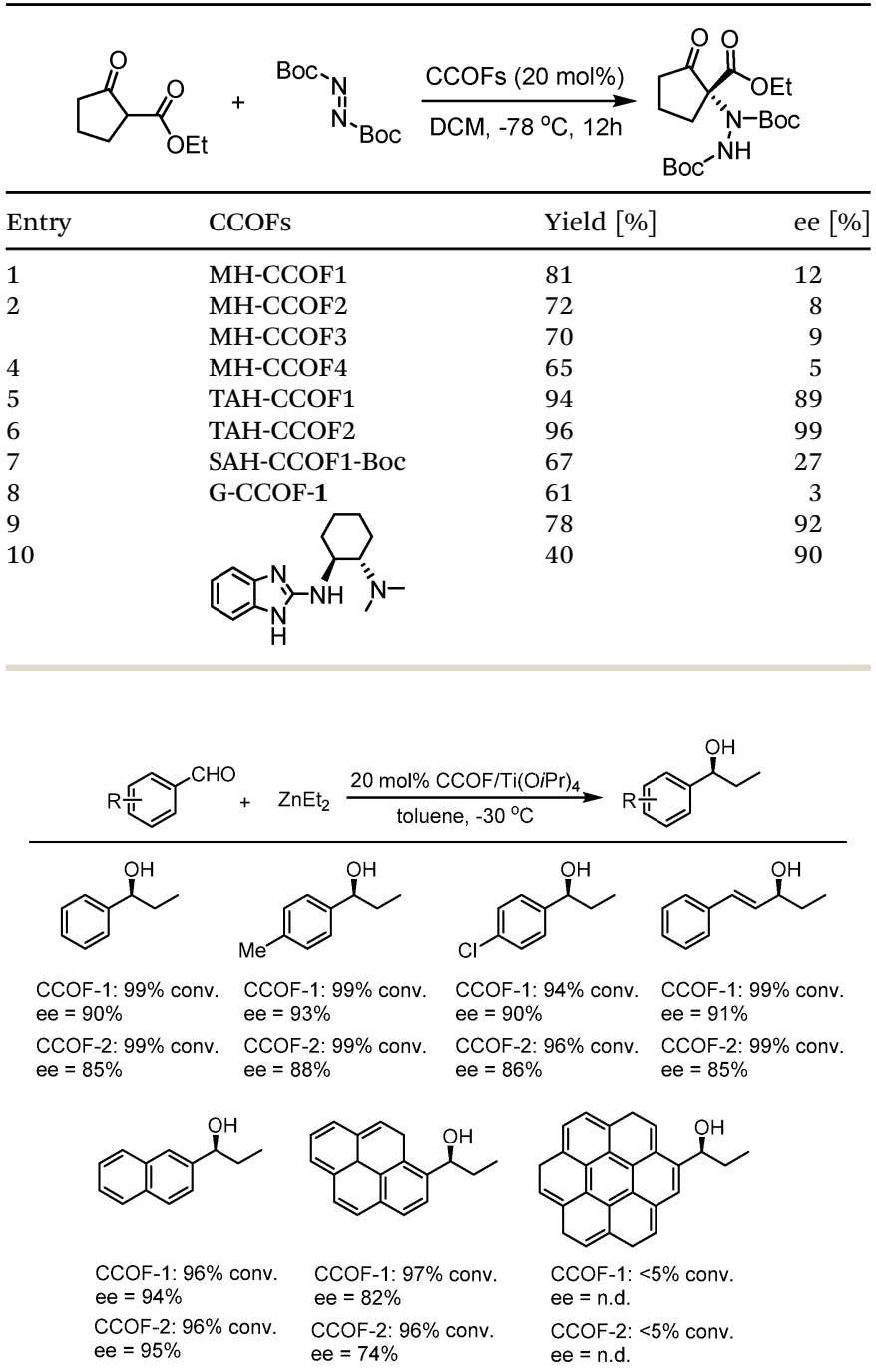

Fig. 18 Addition of diethylzinc to aromatic aldehydes catalyzed by CCOF/Ti.

was detected, indicating that reactions for other aldehydes mainly occur within the CCOFs. ${ }^{91}$

$N, N^{\prime}$-Bis(salicylidene)ethylenediamine (salen) is recognized as one of the most important ligands in coordination chemistry and many metal-salen complexes are well-known privileged ligands for catalysis. ${ }^{152,153}$ Construction of salen-based materials such as silicas, ${ }^{154}$ polymers ${ }^{155}$ and metal-organic frameworks $^{117,156,157}$ has been developed for decades. Wang et al. utilized achiral salen ligands as building blocks to construct COF materials for efficiently catalyzing the Henry reaction ${ }^{121}$ and Yang et al. reported the synthesis of a salen-based achiral COF for cycloaddition reactions of epoxides with $\mathrm{CO}_{2}{ }^{122}$

After exchanging $\mathrm{Zn}^{2+}$ ions for other metal ions of $\mathrm{Zn}$ (salen)based CCOFs (CCOF 4), the M(salen)-based CCOFs (4-M, $\mathrm{Mn}^{+}=$ $\left.\mathrm{Cr}^{2+}, \mathrm{Co}^{2+}, \mathrm{Mn}^{2+}, \mathrm{Fe}^{2+}, \mathrm{V}^{4+}\right)$ are proved to be efficient heterogeneous catalysts for asymmetric cyanation of aldehydes, DielsAlder reaction, alkene epoxidation, epoxide ring-opening, and related sequential reactions. The $\mathbf{4}-\mathbf{V}$ promoted cyanation of 


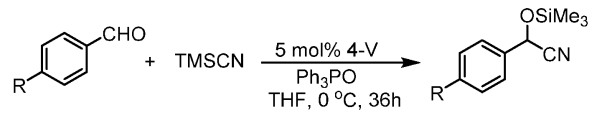

$77-79 \%$ conv.; $89 \%$ ee $(R=M e), 89 \%$ ee $(R=B r), 94 \%$ ee $(R=O M e)$

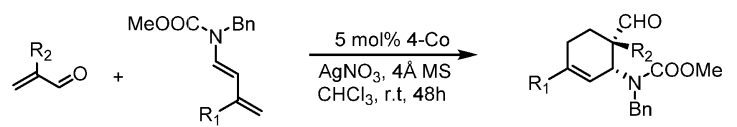

$83-88 \%$ conv.; $86 \%$ ee $\left(R_{1} / R_{2}=H / M e\right), 89 \%$ ee $\left(R_{1} / R_{2}=H / E t\right), 96 \%$ ee $\left(R_{1} / R_{2}=M e / E t\right)$
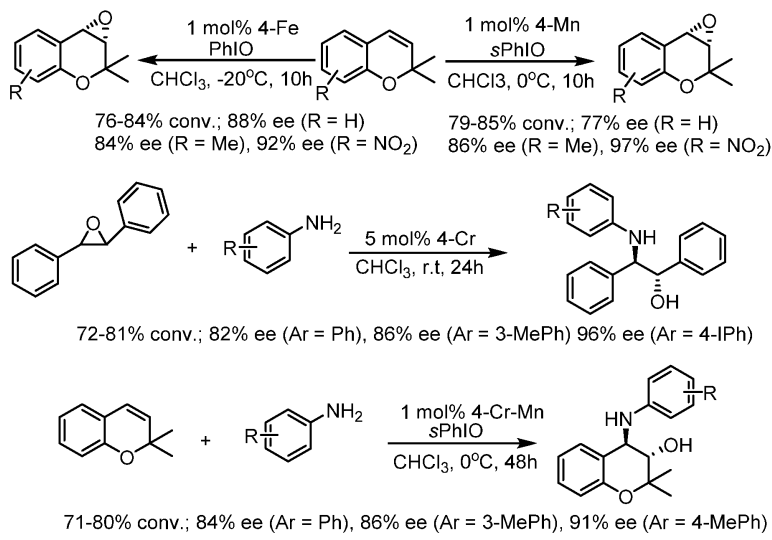

Fig. 19 Asymmetric reactions catalyzed by M(salen)-based CCOFs.

aldehydes with TMSCN gave $89-94 \%$ ee of cyanohydrin silyl ethers. The DA reactions promoted by 4-Co provided the cycloadducts with $86-96 \%$ ee; 4-Fe promoted the oxidation reactions with up to $92 \%$ ee; and $\mathbf{4 - C r}$ catalyzed the aminolysis reactions affording $82-96 \%$ ee of the amino alcohols. Besides, 4-Cr-Mn bearing two different active metal centers could be used for the synthesis of complex molecules with good stereoselectivity in the sequential reactions, by epoxidation of alkene followed by ring-opening of epoxide to afford the amino alcohol with up to $91 \%$ ee (Fig. 19). All CCOF catalysts can be recycled and reused at least 5 times with crystallinity retained and without obvious loss of activity and enantioselectivity. ${ }^{85}$

Cui et al. developed a multivariate strategy for the construction of two- and three-component 2D CCOFs with mixtures of triamines with and without chiral organocatalysts and with a dialdehyde in 2017. Controlled crystallinity and stability were realized and the ternary CCOFs could be used as efficient heterogeneous catalysts for an asymmetric aminooxylation reaction, an aldol reaction, and the Diels-Alder reaction. Four asymmetric organocatalysts L-proline- and L-imidazolidine-based TPB derivatives as chiral knots and DMTA as linkers were used to prepare multivariate CCOFs $9^{\prime}-12^{\prime} / \mathrm{TPB}$. The optimized ternary CCOFs $9^{\prime}-11^{\prime} /$ TPB could promote $\alpha$-aminooxylation reactions between aldehydes and nitrosobenzene affording $\alpha$-aminooxy aldehydes in $75-77 \%$ isolated yield with up to $95 \%$ ee. The selected ternary CCOFs could also catalyze the aldol reaction of cyclohexanone with 4-nitrobenzaldehyde and 3-nitrobenzaldehyde, affording the products in 92 and 86\% ee, 95 and 94\% yield, and 90:10 and 90:10 anti/syn ratio. Furthermore, the imidazolidinonecontaining ternary CCOFs $12^{\prime} /$ TPB could promote the asymmetric Diels-Alder cycloaddition reaction of cyclopentadiene and aldehydes with different substituents to afford the cycloadduct
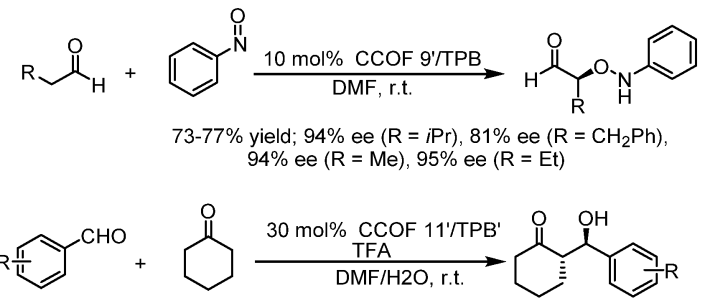

$85-95 \%$ yield; $9: 1$ anti/syn and $92 \%$ ee $\left(\mathrm{R}=4-\mathrm{NO}_{2}\right)$ 9:1 anti/syn and $86 \%$ ee $\left(\mathrm{R}=3-\mathrm{NO}_{2}\right)$

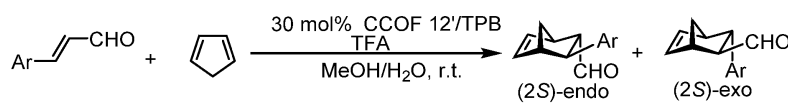

$76-85 \%$ yield; $13: 1$ endo/exo and $90 \%$ ee for the major isomer $(\mathrm{Ar}=\mathrm{Ph})$

$1: 5$ endo/exo and $95 \%$ ee for the major isomer $(\mathrm{Ar}=4-\mathrm{BrPh})$

$1: 7$ endo/exo and $95 \%$ ee for the major isomer $(\mathrm{Ar}=4-\mathrm{MeOPh})$

$1: 22$ endo/exo and $90 \%$ ee for the major isomer $\left(\mathrm{Ar}=4-\mathrm{NO}_{2} \mathrm{Ph}\right)$

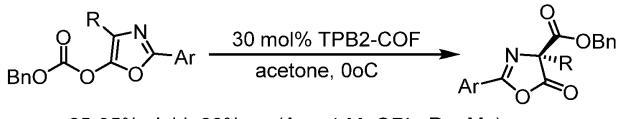

$85-95 \%$ yield; $80 \%$ ee $(\mathrm{Ar}=4-\mathrm{MeOPh}, \mathrm{R}=\mathrm{Me})$ $83 \%$ ee $\left(\mathrm{Ar}=4-\mathrm{MeOPh}, \mathrm{R}=\mathrm{CH}_{2} \mathrm{CH}_{2} \mathrm{SCH}_{3}\right)$

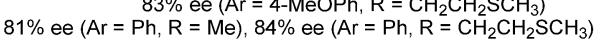

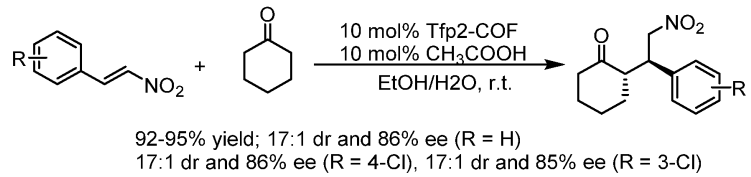

Fig. 20 Asymmetric $\alpha$-aminooxylation of aldehydes, Aldol reactions, Diels-Alder reactions, Steglich rearrangement and Michael addition reactions catalyzed by optimized CCOFs.

in $76-85 \%$ isolated yield and high selectivity (90-95\% ee for the major exo isomer, 5:1-22:1 exo/endo). These COF catalyzers could be recycled and reused at least five times without an obvious loss of activity and enantioselectivity with crystallinity and porosity maintained. ${ }^{29}$ In 2019, this research group also reported two DHIP- and pyrrolidine-based 2D CCOFs by directly employing chiral functionalities as building units, and the as-synthesized CCOFs could serve as effective and recyclable heterogeneous catalysts for asymmetric Steglich rearrangement and asymmetric Michael addition, displaying high enantioselectivity and diastereoselectivity with up to $84 \%$ ee value for Steglich rearrangement and 86\% ee value, 17:1 dr value for the Michael addition reaction (Fig. 20). ${ }^{81}$

Other types of CCOFs for the application of asymmetric catalysis have also been reported in recent years. After postsynthetic modification of the enaminone groups of CCOF with $\mathrm{Cu}(\mathrm{II})$ ions, the material CCOF-TpTab-Cu was a recyclable heterogeneous catalyst for the asymmetric Henry reaction of nitroalkane with aldehydes with moderate ee values (35\%) (Fig. 21). ${ }^{8}$ The same year, Ma et al. reported a series of COFenzyme composites with enhanced enzymatic performance; the resulting biocomposites efficiently catalyzed the kinetic resolution of secondary alcohols with vinyl acetate with ee values higher than $99.5 \% .{ }^{158}$ In 2019, Cui et al. synthesized chiral COF and MOF based materials by combining COF/MOF with homogeneous linear chiral copolymers to construct hybrid stereoselective catalysts. The resulting chiral polymer/COF (MOF) 


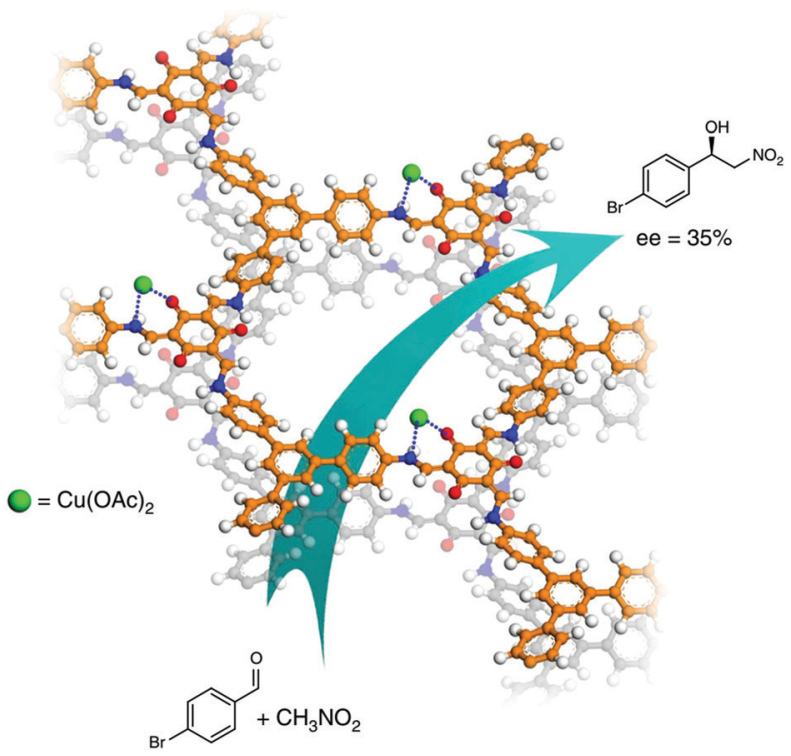

Fig. 21 Henry reaction between nitroalkane and aldehyde catalyzed by CCOF-TpTab-Cu. Adapted from ref. 88 with permission from Nature Research, copyright 2018.

composite materials treated as heterogeneous catalyst revealed good enantioselectivity and diastereoselectivity for the direct asymmetric aldol reactions with up to $99 \%$ ee and $96: 4 \mathrm{dr}^{159}$

Utilizing the advantages of the chiral functionalities and the loaded highly active Pd NPs, Dong et al. employed Pd@CCOFMPC for the asymmetric Henry reaction and asymmetric reductive Heck reaction. For the chosen Henry reaction (nitroaldol reaction of benzaldehyde with nitromethane), Pd@CCOF-MPC exhibited good catalytic activity and enantioselectivity, which is demonstrated by the milder reaction conditions (r.t. and short reaction time) with up to $99 \%$ yield and $97 \%$ ee value. For the asymmetric reductive Heck reaction of 2-cyclohexen-1-one with bromobenzene, Pd@CCOF-MPC also showed high activity and selectivity in that the yield reached $99 \%$ with $94 \%$ ee value. This work highlights the potential of the preparation of new asymmetric catalysts by combining CCOF supports and metal NPs (Fig. 22). ${ }^{82}$

Another CCOF synthesized by using $S$-(+)-2-methylpiperazine was reported by Dong et al. in 2019. The photothermal conversion species of copper tetrabromophenolphthalein is another cross-linker and a porphyrin-containing homochiral COF, CCOF-CuTPP, was obtained. Very similar to their last CCOF work, metal nanoparticles (M NPs) were impregnated into CCOFs via successive solution impregnating and metal reduction steps, and two M@CCOF-CuTPP $(\mathrm{M}=\mathrm{Au}$, Pd) were constructed. CCOF-CuTPP is a rigid homochiral hostframework that can provide a powerful chiral confined space even at high temperatures. Moreover, the porphyrin moiety can readily convert light into thermal energy. Thus, after metal nanoparticles are loaded into the porous and heteroatom-rich CCOF, Au@CCOF-CuTPP and Pd@CCOF-CuTPP feature chiral templating, photothermal conversion and high catalytic activity, which make them good catalyzers for thermally driven asymmetric

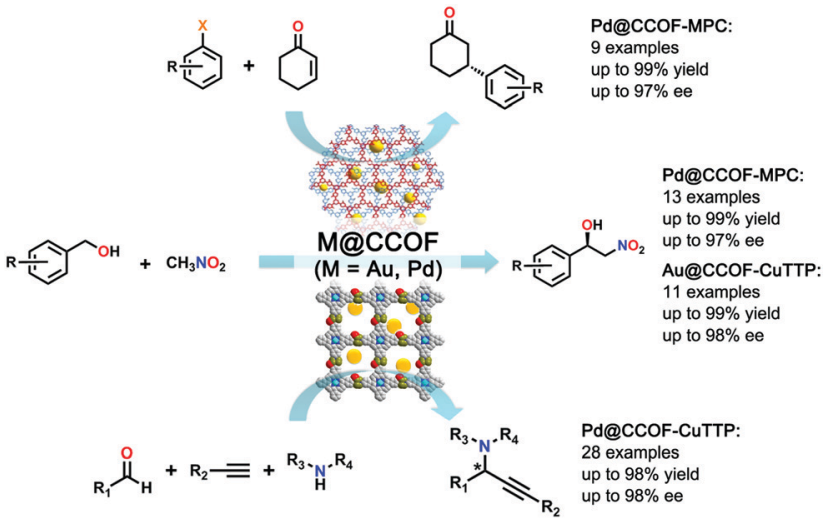

Fig. 22 Asymmetric Henry reaction, reductive Heck reaction and $\mathrm{A}^{3}$-coupling reactions catalyzed by MaCCOF $(M=A u, P d)$. Reproduced from ref. 82 with permission from the American Chemical Society, copyright 2017. Reproduced from ref. 83 with permission from Nature Research, copyright 2019

one-pot Henry and $\mathrm{A}^{3}$-coupling reactions with both high yield (up to $99 \%$ yield) and stereoselectivity (up to $98 \%$ ee value) (Fig. 22 ). ${ }^{83}$

\subsection{Chiral separation}

Utilizing the well-defined and precise pore structure of multifunctional COFs, they can be applied in separation technology like gas separation, ${ }^{160}$ water treatment (desalination and dye removal), ${ }^{161}$ organic solvent nanofiltration (OSN) ${ }^{162}$ and pervaporation. ${ }^{163}$ Most COF-based separation is based on size exclusion like membrane separation. ${ }^{164-166}$ Nevertheless, this field still faces huge challenges and opportunities. ${ }^{34}$

Separation of enantiomers is of great significance in the field of pharmacology and biology, because pure isomers may have different properties in biological interactions, and pharmacology and toxicology ${ }^{167-169}$ Chromatographic techniques based on chiral stationary phases are considered to be one of the most effective methods to separate and obtain enantiopure compounds. ${ }^{170-172}$ Recently, new chiral porous materials such as metal-organic frameworks, ${ }^{173}$ porous organic frameworks ${ }^{91}$ and porous organic cages ${ }^{174}$ as a chiral stationary phase have attracted lots of attention. The CCOF as a stationary phase for chiral separation has great potential and application prospects.

In 2016, Yan et al. reported the first application of CCOF materials for chiral gas chromatography. The CCOFs were functionalized with chiral (+)-diacetyl---tartaric, and the chiral COF-bound capillary columns were fabricated utilizing the in situ growth approach and could realize the baseline separation of enantiomers such as ( \pm )-1-phenylethanol, $( \pm)$-1-phenyl1-propanol, $( \pm)$-limonene and $( \pm)$-methyl lactate within $5 \mathrm{~min}$. The (+)-enantiomers were more ordered in the microenvironment of the chiral COFs with stronger interaction than the (-)-enantiomers. Furthermore, adsorption enthalpy between the framework and each enantiomer were measured. Higher adsorption enthalpy of between the COF and (+)-enantiomers suggest that the high-resolution chiral separation is driven by enthalpy. The CTpPa-1-bound capillary column also exhibits excellent repeatability and recyclability; the relative standard 
a)

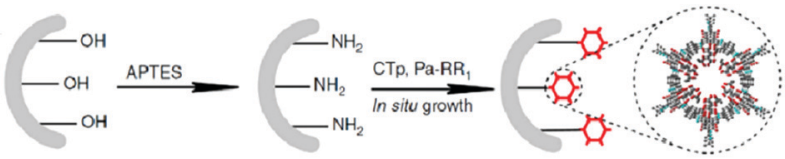

b)
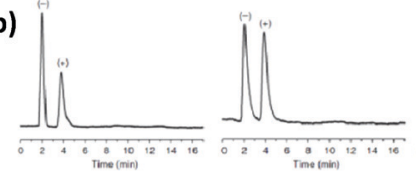

( \pm )-1-phenylethano

$(+1+1$

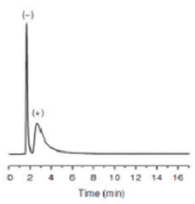

( \pm )-methyl lactate

Fig. 23 (a) The in situ growth approach by injecting a solution of CTp and Pa-1 into the amino-modified capillary column. (b) Gas chromatograms of ( \pm )-1-phenylethanol, $( \pm)$-1-phenyl-1-propanol, $( \pm)$-limonene and $( \pm)$-methyl lactate on a CTpPa-1-bound capillary column. Adapted from ref. 28 with permission from Nature Research, copyright 2016.

deviation of the retention time for the run to run $(n=7)$, day to day $(n=5)$ and column to column $(n=3)$ was $0.15-0.30 \%$, $1.11-1.89 \%$ and $2.35-3.41 \%$, respectively (Fig. 23 ). ${ }^{28}$

COFs could also be a good candidate to be used for the separation of small molecules in high performance liquid chromatography (HPLC). ${ }^{79,175,176}$ In 2018, two 3D CCOF (CCOF 5 and 6) packed columns for HPLC were prepared by loading the mixture of the crystalline samples and silica. As chiral stationary phases (CSPs) for HPLC, they could separate racemic alcohols, sulfoxides, carboxylic acids and esters. The performance of the column packed with CCOF 5 was evaluated by resolution of 1-phenyl-2-propanol, 1-phenyl-1-pentanol, 1-phenyl-1-propanol and 1-(4-bromophenyl)-ethanol with good selectivity factor and chromatographic resolution, affording $\alpha / R_{\mathrm{S}}=1.19 / 1.52,1.20 / 0.92,1.21 / 1.26$ and $1.17 / 1.44$, respectively. Oxidation of imine linkages of CCOF 5 affords a stability-enhanced

amide-linked framework (CCOF 6). Baseline separation of racemates of 1-phenyl-2-propanol, 1-phenyl-1-pentanol, 1-phenyl-1propanol and 1-(4-bromophenyl)-ethanol can be achieved by the column packed with 6 , affording $\alpha / R_{\mathrm{S}}=1.29 / 1.78,1.21 / 1.58$, $1.33 / 2.47$ and 1.24/1.54, respectively (Fig. 24).

The pore interior of the oxidized CCOF was fine-tuned and CCOF 6 showed superior resolution performance compared with the pristine framework. Other types of racemates such as sulfoxides, carboxylic acids and esters can also be completely or partially resolved on the COF-based CSPs. Both COFs can work as CSPs of HPLC for separation of racemic alcohols with excellent durability. Separation performances remained the same after repeatability testing for at least 2 months. The chiral separation ability as CSPs of amorphous COF@SiO ${ }_{2}$ and $(R, R)$ $\mathrm{TTA} / \mathrm{SiO}_{2}$ hybrid microspheres was also evaluated for comparison. The results showed that they cannot separate racemic alcohols, further highlighting the key role of the crystalline 3D structure of the COF in chiral separation. This work advances COFs as a platform for chiral resolution and will expand the scope of materials design and engineering to make new types of COFs with unique enantioselective functions (Fig. 24). ${ }^{86}$

In 2018, Ma et al. reported a series of COFs with enriched chirality (biomolecules $\subset \mathrm{COFs}$ ) by covalently immobilizing biomolecules (amino acids, peptides, enzymes) into polyimide achiral COFs. The as-synthesized lysozyme $\subset$ COF 1 was employed as chiral stationary phases for separating various racemates including DL-tryptophan, DL-leucine, DL-threonine, DL-lysine, DL-aspartic acid, ofloxacin, (+/-)-propranolol hydrochloride, metoprolol tartrate, alanyl glutamine, chlorpheniramine, and benzoin. High chiral separation efficiencies are found for both normal and reverse phases in high-performance liquid chromatography (HPLC). Besides, lysozyme $\subset$ COF $1 \mathrm{CSP}$ is also confirmed to

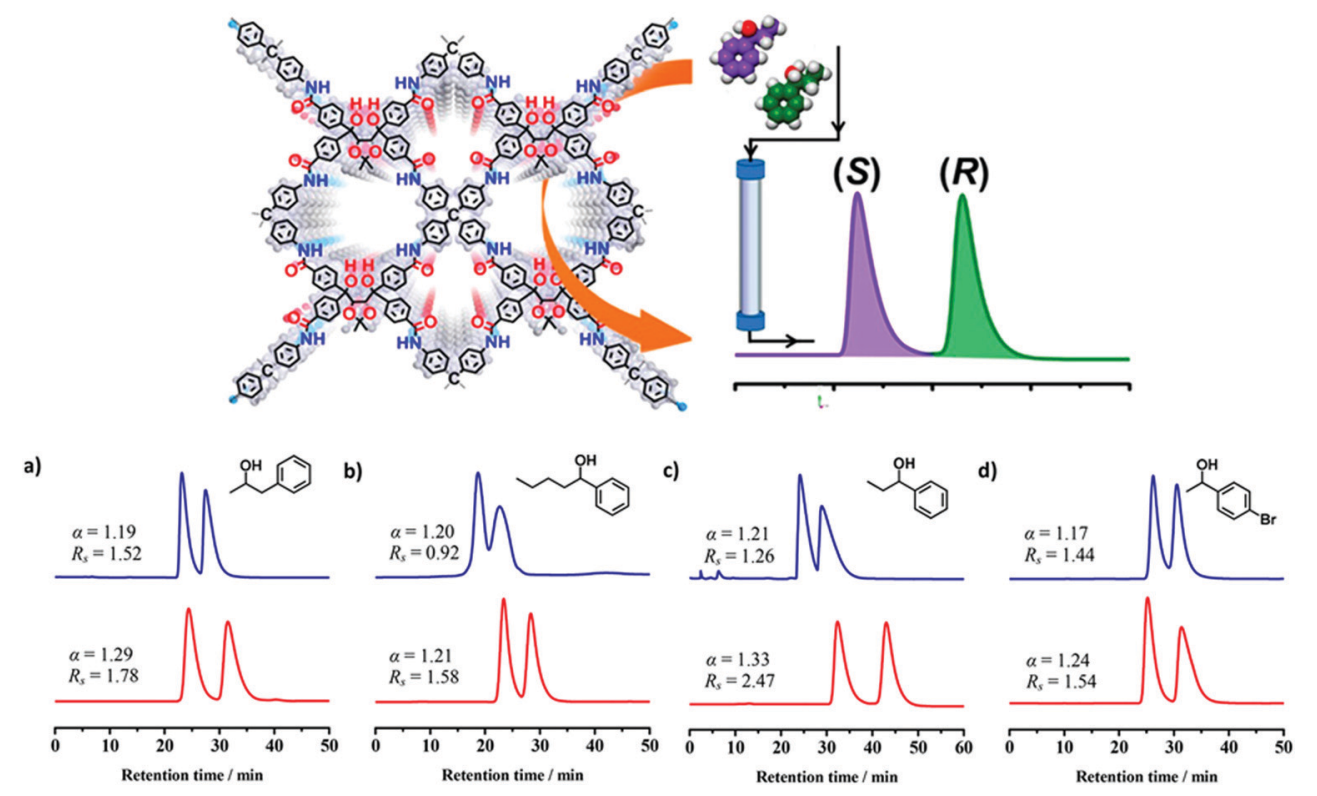

Fig. 24 (a-d) HPLC separation chromatograms of racemic 1-phenyl-2-propanol, 1-phenyl-1-pentanol, 1-phenyl-1-propanol and 1-(4-bromophenyl)ethanol on the CCOF 5 (blue line) and 6 (red line) packed columns. Reproduced from ref. 86 with permission from the American Chemical Society, copyright 2018. 

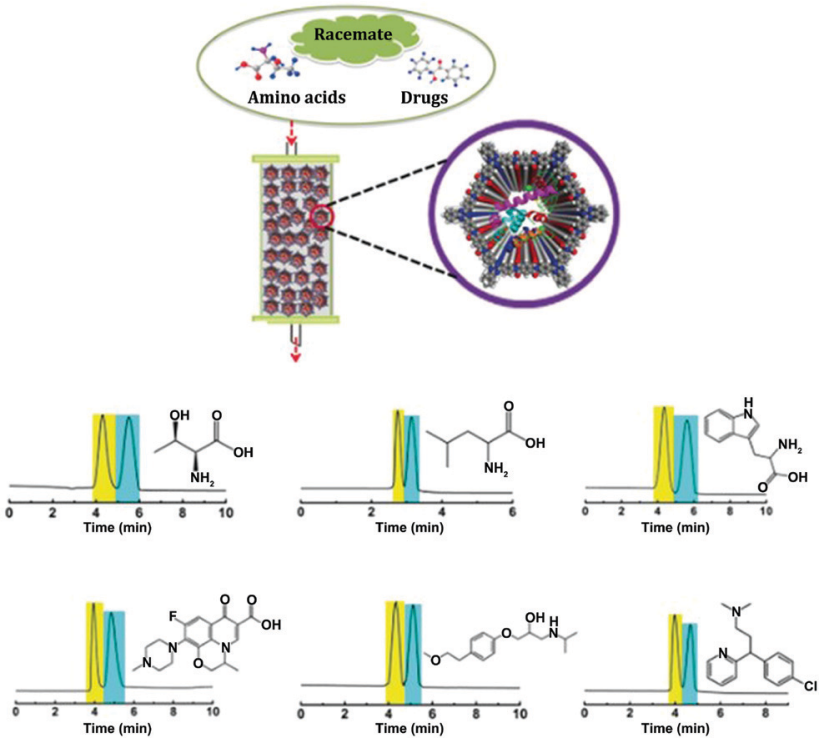

Fig. 25 (a) Illustration of lysozyme $\subset$ COF 1 based CSPs for chiral separation; (b-g) HPLC separation chromatograms of DL-threonine, DL-leucine, DL-tryptophan, ofloxacin, metoprolol, and chlorpheniramine. Reproduced from ref. 74 with permission from Wiley, copyright 2018.

have good repeatability and reusability for $>120$ separation runs during the tested period ( 2 months) with high efficiency maintained (Fig. 25). ${ }^{74}$

\subsection{Chiral recognition}

Recently, similar to other porous materials like MOFs, ${ }^{177} \mathrm{COF}$ materials have also shown great potential as sensors. COFs with large $\pi$-conjugated systems within and/or between building units often exhibit intense fluorescence emissions. On the other hand, one can easily engineer COF structures or functions to have strong interaction towards an analyte, including biomolecules. Demonstrated applications of COFs include explosive sensing, ${ }^{178}$ humidity sensing, ${ }^{179} \mathrm{pH}$ detection, ${ }^{180}$ biosensing, ${ }^{181}$ gas sensing, ${ }^{182}$ and metal ion sensing. ${ }^{183}$ For example, in 2016, Jiang et al. developed a highly luminescent AIE-active COF as a highly sensitive sensor to detect ammonia $(\mathrm{AIE}=$ aggregation-induced emission $) .{ }^{54}$ Wang et al. constructed a thioether-functionalized COF for highly sensitive detection and effective removal of $\mathrm{Hg}^{2+} \cdot{ }^{184}$ In addition, recognition of chiral biomolecules such as saccharides and amino acids is an exciting area for COF research. Inspiration can be drawn from the development in supramolecular chemistry. ${ }^{185}$

For example, CCOFs with Tp monomers are highly emissive. Enantioselective sensing of saccharides was therefore examined using CCOF TpTab. The emission at $540 \mathrm{~nm}$ was decreased when TpTab was treated with aliquots of D-cellobiose, but the rate of change with (4)-TpTab was faster than that with (4)-TpTab. SternVölmer (SV) behavior in the $0-7 \times 10^{-5} \mathrm{mM}$ concentration range of disaccharide quenchers was studied. The association constants $\left(K_{\text {SV }}\right)$ were calculated as $13,086 \pm 1000 \mathrm{M}^{-1}$ with $(\Lambda)$-TpTab and $3806 \pm 200 \mathrm{M}^{-1}$ with (4)-TpTab. A quenching ratio [QR = $\left.K_{\mathrm{Sv}}(\Lambda \mathrm{TpTab}) / K_{\mathrm{Sv}}(\Delta-\mathrm{TpTab})\right]$ of $3.44 \pm 0.26: 1$ was obtained (Fig. 26$)$. a)

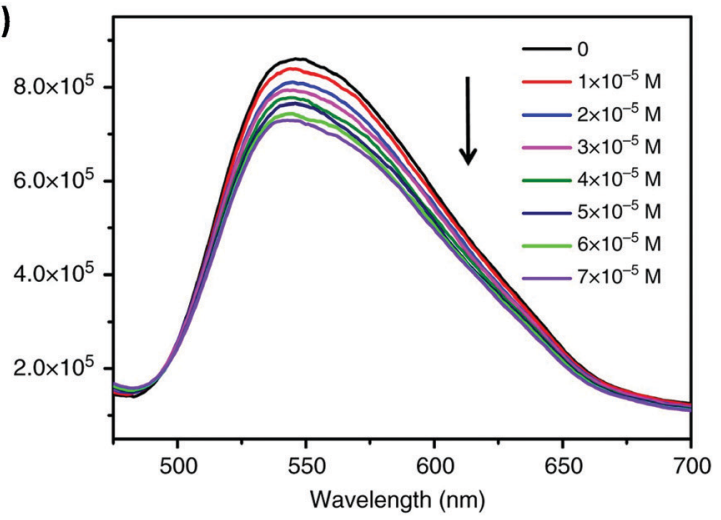

b)

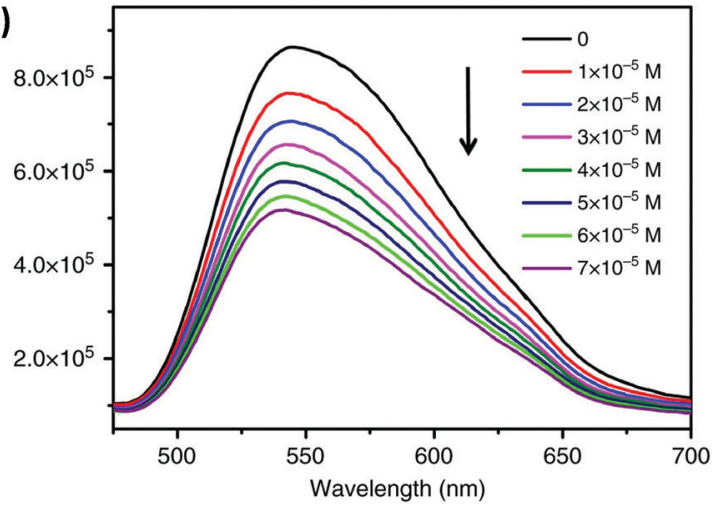

c)

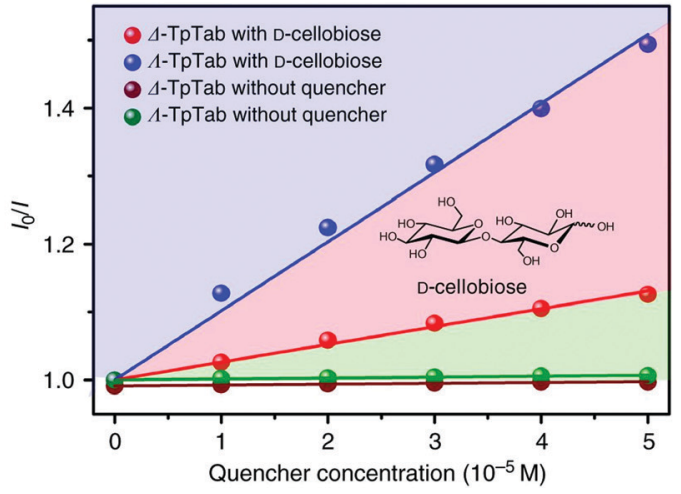

Fig. 26 ( $a$ and b) The fluorescence emission spectra of the $(\Lambda) /(\Delta)-T p T a b$ COF with increasing concentration of the D-cellobiose quencher. (c) SV plots of the fluorescence emissions of the $(\Lambda) /(\Delta)-T p T a b$ COF quenched by $\mathrm{D}$-cellobiose. Adapted from ref. 88 with permission from Nature Research, copyright 2018.

The quenching of TpTab with different amounts of disaccharide was highly efficient, and the fluorescence quenching is likely due to the supramolecular interactions of TpTab with saccharides, forming a CCOF-saccharide adduct. Furthermore, the CCOF TpTab showed high enantioselectivity toward chiral carbohydrates including saccharides, including D-glucose, D-mannitol, D-sucrose, D-lactose, D-maltose, D-sorbitol, D-fructose, D-gentiobiose, D-lactobionic acid, D-glucuronic acid, and D-gluconic acid. Fluorescence quenching $\mathrm{QR}$ values were determined as $1.56 \pm 0.2,2.18 \pm 0.4,2.43 \pm 0.16,1.59 \pm 0.25,3.62 \pm$ $0.16,2.24 \pm 0.19,2.49 \pm 0.21,2.66 \pm 0.1,2.00 \pm 0.30,1.32 \pm$ 0.41 , and $2.03 \pm 0.28$, respectively. ${ }^{88}$ 
Chiral discrimination of vapours is crucial for olfactory perception in biological systems. This inspires the development of artificial chiral vapour sensors. ${ }^{186}$ However, research in this direction remains a big challenge. For the first time, in 2019, Cui et al. reported two Binol-based CCOFs that can recognize chiral vapours. The as-synthesized CCOFs could be easily exfoliated into ultrathin 2D nanosheets (NSs) (denoted as 7-NS) due to the twisted TPE species by solvent-assisted liquid sonication. Free-standing nanofiber membranes (denoted as 7@PVDF) were fabricated by electrospinning. The fluorescence of 7-NS and 7@PVDF was examined for fluorescence recognition and sensing of chiral vapors including $\alpha$-pinene, limonene, fenchone, carvone, and terpinen-4-ol was performed. The results showed that fluorescence emissions could be effectively quenched by these chiral vapours. When 7-NS or 7@PVDF was treated with the two enantiomers of $(\alpha)$-pinene, the (-)-enantiomers displayed faster quenching rates than the (+)-enantiomers. Furthermore, for enantioselective quenching ratios of several terpenes, both 7-NS and 7@PVDF showed higher enantioselectivity than bulk CCOFs under the same conditions (Fig. 27). The 7@PVDF membrane could be directly reused for 3 cycles of sensing without significant loss of enantioselectivity and the structure remained almost unchanged. The CCOF NSs exhibited superior enantioselectivity of chiral sensing compared to the bulk CCOF-based systems, likely due

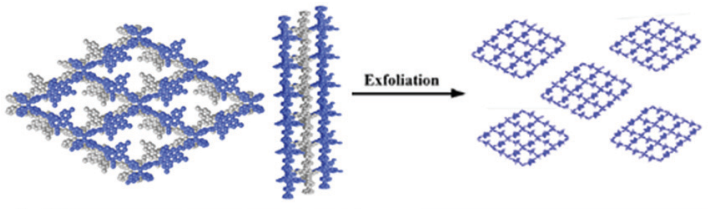

a)

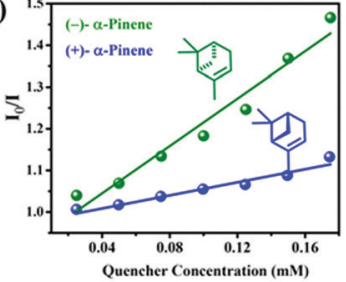

c)
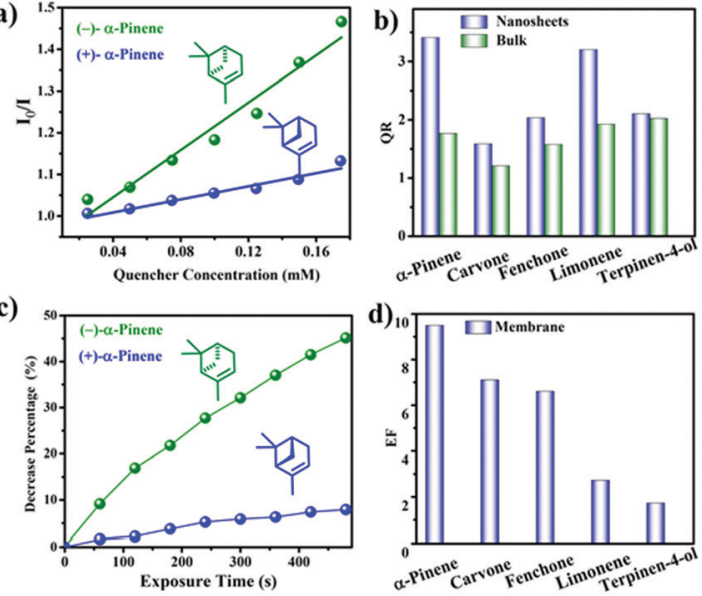

e)

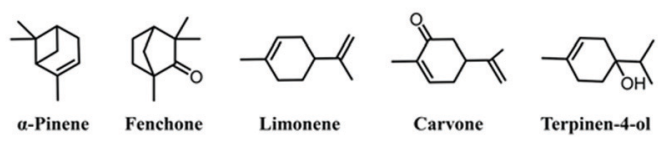

Fig. 27 The illustration of CCOF 7 exfoliation and the applications in fluorescence recognition. (a) Stern-Volmer plots of 7-NS upon titration of $\alpha$-pinene in MeCN. (b) Enantioselective quenching ratio for several terpenes. (c) Decrease percentage upon exposure to $\alpha$-pinene for 7aPVDF. (d) Enantioselective fluorescence decrease ratio for several vapors. (e) Chemical structures of terpenes. Reproduced from ref. 87 with permission from the American Chemical Society, copyright 2019. to steric confinement of the CCOF channels that create a discriminating chiral environment and conformational rigidity arising from BINOL groups. ${ }^{87}$

Transmembrane molecules and ion channels in the nanoscale are widely found in biological systems and are crucially important for a variety of physiological activities. ${ }^{187-189}$ Notably, 2D CCOFs tend to form uniform and controllable chiral 1D mesochannels by the stacking of monolayers. ${ }^{190-192}$ The nanochannels of 2D COFs may offer predesigned transport pathways for ions and molecules. In 2019, Cui, Long and co-workers reported utilizing nanochannels of 2D CCOFs for enantioselective transmembrane transport of amino acids (Fig. 26). Two vinyl-functionalized 2D COFs were crystallized, and after the post-modification of $\beta$-cyclodextrin $(\beta-\mathrm{CD})$, the CD-COFs were further fabricated into free-standing mixed matrix membranes (MMMs) that can selectively transport amino acids. The chiral recognition ability was monitored by both transmembrane ionic current measurements and permeation experiments driven by osmotic pressure. After the CD-COF MMM was exposed to $\mathrm{L}^{-}$or $\mathrm{D}^{-h i s t i d i n e, ~ t h e ~ t r a n s m e m b r a n e ~}$ ionic currents increased only for L-histidine while almost no changes were observed for D-histidine. To evaluate the change, they calculated the current change ratio $\left(R=\left(I-I_{0}\right) / I_{0}\right.$, where $I_{0}$ and $I$ are the current measured at $-1 \mathrm{~V}$ before and after treatment with His). The $R_{\mathrm{L}-\mathrm{His}}$ and $R_{\mathrm{D}-\mathrm{His}}$ values were 0.34 and 0.01 for the CD-COF-1 MMM, respectively. The change in the $I-V$ characteristics could confirm the selective binding of L-histidine into the CCOF nanochannels. Moreover, the $\beta$-CDCOF channel provides a pathway for $\mathrm{L}$-His transport across the membrane. The CD-COF MMM was fixed tightly in a cell at room temperature. In one chamber of the cell, the $\mathrm{D}$ - or L-histidine buffer solution was used as a feed solution, and the concentrations of permeated solute at the other side of the cell were measured by HPLC. The flux $(J)$ of permeation was calculated according to the equation $J=C / A t$, where $C$ is the concentration of permeated solution, $t$ is the permeation time, and $A$ is the effective membrane area. The $J_{\mathrm{L}-\mathrm{His}}$ of the CD-COF-1 MMM was calculated to be $1.04 \mu \mathrm{M} \mathrm{cm}^{-2} \mathrm{~h}^{-1}$ while the $J_{\mathrm{D}-\mathrm{His}}$ was $0 \mu \mathrm{M} \mathrm{cm}^{-2} \mathrm{~h}^{-1}$. This indicated that the penetration rate of $\mathrm{L}$-His was much faster than that of D-His (Fig. 28). This could be attributed to the enantioselective adsorption and desorption of $\mathrm{L}$-histidine by the $\beta$-CD-COF nanochannels. This work paves a way for COF nanochannels as a platform with chiral functional groups for selective transmembrane transport of small molecules and even biomolecules. ${ }^{76}$

\section{Conclusions and outlooks}

The development of homochiral COFs is still in its infancy. Nevertheless, tremendous progress is achieved. In this review, we summarized the developments of CCOFs including the synthesis strategies, peculiar structures and their applications in heterogeneous asymmetric catalysis, enantioselective separation, and chiral recognition and sensing.

With the help of three synthetic strategies (post-synthesis, direct synthesis, and chiral induction synthesis), several crystalline 
a)
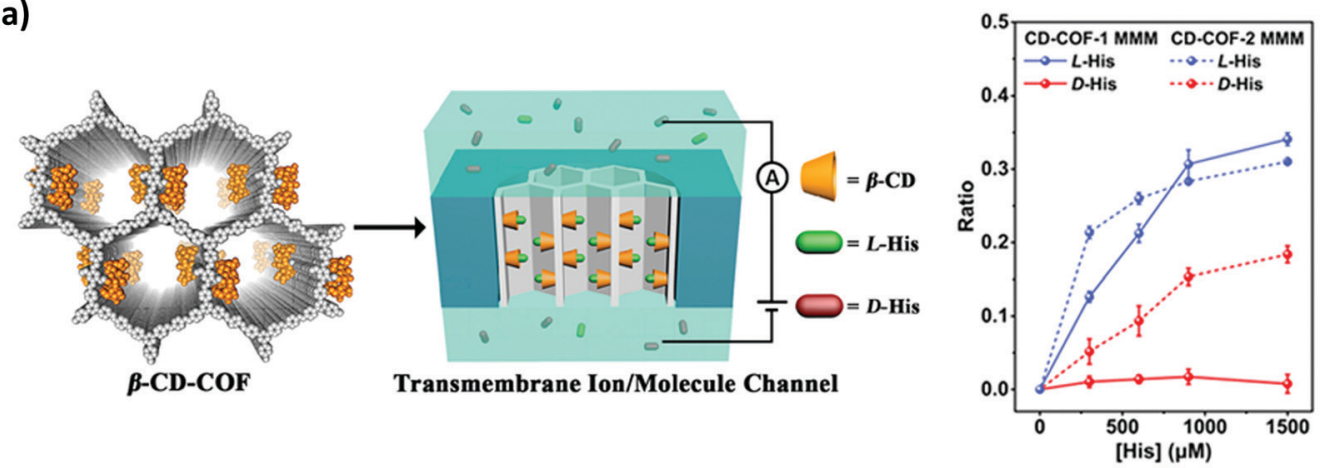

b)
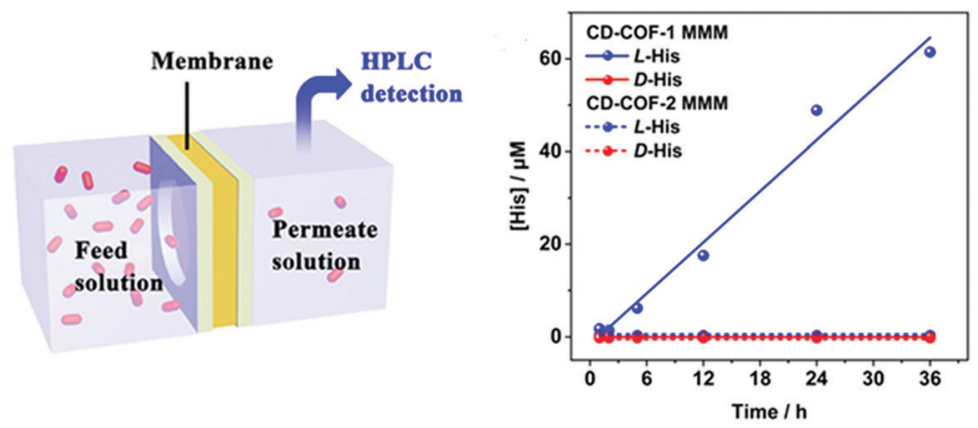

Fig. 28 (a) The illustration of the ionic current experiment of the CD-COF-MMM system and the ionic current change ratio versus the concentration of histidine. (b) The illustration of the transmembrane transport experiment and the concentration of permeate solution at different permeation times. Reproduced from ref. 76 with permission from the American Chemical Society, copyright 2019.

chiral COFs have been carefully customized. Among these strategies, direct synthesis of CCOF, especially by using chiral skeleton monomers, is advantageous in producing CCOFs with open channels, permanent porosity, highly populated chiral centres, and well-defined structures. However, challenges still remain in synthesizing new CCOFs. Development of synthetic methods for CCOFs is needed to balance the crystallographic symmetry and the asymmetry arising from chiral functionalities. As such, methods beyond solvothermal synthesis, including microwave-assisted reactions, room-temperature synthesis, or chemosynthesis, might be worth exploring.

In addition to developing synthetic methods, vast opportunities lie in diversifying linkages. Almost all homochiral COFs reported to date are synthesized through condensing amines and aldehydes. The resulting imine bonds have shown satisfactory hydrolytic stability. However, to improve their stability towards acid or basic conditions, one might consider other linkages such as olefin, phenazine, imide, or dioxin. Other linkages that can foster extended conjugated systems might give rise to interesting electronic properties. These properties might in turn provide opportunities for chiral recognition and sensing to read out using electrical signals.

Owing to the unique structural features and facile tunability of the subcomponents and channels, CCOFs show great potential in heterogeneous catalysis, enantioselective separation, and recognition. Some typical asymmetric reactions, such as Aldol reaction, Henry reaction, and Diels-Alder reaction, have been achieved by CCOF-based catalysts successfully, but more complex and cutting-edge organic reactions remain to be developed. Besides, compared to other porous materials, the advantages of COFs in the field of asymmetric catalysis should be explored in the future. In addition, the fundamental mechanism of the COF-based chiral separation and recognition system still remains unclear. Theoretical calculation as a very useful tool is capable of predicting the structures and properties as well as simulating the separation and recognition process. As such, the crystalline processes of CCOFs should also be studied in more detail to prepare single crystals of CCOFs, which can provide more accurate structural information at the atom level via single-crystal X-ray diffraction analysis. The structural studies of CCOFs would not only provide more direct evidence of the mechanisms in catalysis and recognition processes, but also help us to understand the origin, transfer, and amplification of the chirality in the porous framework. Furthermore, with a judicious choice of chiral building blocks, more functional 2D and 3D CCOFs, particularly those involving "privileged" chiral organic linkers, chiral organic catalysts and bioactive molecules, that can satisfy the requirements for different applications could be expected in the near future. The rational design and controllable synthesis of stable and highly crystalline CCOFs with tunability in composition and topological structures has become an urgent necessity.

\section{Conflicts of interest}

There are no conflicts to declare. 


\section{Acknowledgements}

This work was financially supported by the National Science Foundation of China (Grants 91956124, 21620102001, 21875136, 91856204, 21978058 and 21676094), Key Project of Basic Research of Shanghai (17JC1403100, 18JC1413200 and 19JC1412600), National Key Basic Research Program of China (2016YFA0203400), Shanghai Rising-Star Program (19QA1404300), Postdoctoral Innovative Talent Support Program (BX20190195) and the China postdoctoral science foundation (2019M661483). LL thank University Research Fund Grant (225265) at University of Wellington for support.

\section{Notes and references}

1 S. Weinberg, Phys. Rev., 1968, 166, 1568-1577.

2 G. H. Wagnière, On chirality and the universal asymmetry: reflections on image and mirror image, John Wiley \& Sons, 2008.

3 D. P. Glavin, A. S. Burton, J. E. Elsila, J. C. Aponte and J. P. Dworkin, Chem. Rev., 2019, 120, 4660-4689.

4 W. Ma, L. Xu, A. F. de Moura, X. Wu, H. Kuang, C. Xu and N. A. Kotov, Chem. Rev., 2017, 117, 8041-8093.

5 D. Avnir and D. Huylebrouck, Nexus Netw. J., 2013, 15, 171-182.

6 E. J. Ariens and E. W. Wuis, Clin. Pharmacol. Ther., 1987, 42, 361-363.

7 J. Shen and Y. Okamoto, Chem. Rev., 2015, 116, 1094-1138. 8 A. Calcaterra and I. D'Acquarica, J. Pharm. Biomed. Anal., 2018, 147, 323-340.

9 L. Chen, P. S. Reiss, S. Y. Chong, D. Holden, K. E. Jelfs, T. Hasell, M. A. Little, A. Kewley, M. E. Briggs and A. Stephenson, Nat. Mater., 2014, 13, 954.

10 Novel Drug Approvals for 2019, Retrieved from https:// www.fda.gov/drugs/new-drugs-fda-cders-new-molecularentities-and-new-therapeutic-biological-products/noveldrug-approvals-2019.

11 S. Che, Z. Liu, T. Ohsuna, K. Sakamoto, O. Terasaki and T. Tatsumi, Nature, 2004, 429, 281.

12 C. Li, Catal. Rev., 2004, 46, 419-492.

13 Y. Gao and Z. Tang, Small, 2011, 7, 2133-2146.

14 R. E. Morris and X. Bu, Nat. Chem., 2010, 2, 353-361.

15 C. Tan, D. Chu, X. Tang, Y. Liu, W. Xuan and Y. Cui, Chem. - Eur. J., 2019, 25, 662-672.

16 Y. Liu, W. Xuan and Y. Cui, Adv. Mater., 2010, 22, 4112-4135.

17 K. Gedrich, M. Heitbaum, A. Notzon, I. Senkovska, R. Fröhlich, J. Getzschmann, U. Mueller, F. Glorius and S. Kaskel, Chem. - Eur. J., 2011, 17, 2099-2106.

18 Z. Gu, C. Zhan, J. Zhang and X. Bu, Chem. Soc. Rev., 2016, 45, 3122-3144.

19 A. P. Cote, A. I. Benin, N. W. Ockwig, M. O'keeffe, A. J. Matzger and O. M. Yaghi, Science, 2005, 310, 1166-1170.

20 S. Ding and W. Wang, Chem. Soc. Rev., 2013, 42, 548-568.

21 P. J. Waller, F. Gándara and O. M. Yaghi, Acc. Chem. Res., 2015, 48, 3053-3063.
22 C. S. Diercks and O. M. Yaghi, Science, 2017, 355, eaal1585.

23 X. Wu, X. Han, Y. Liu, Y. Liu and Y. Cui, J. Am. Chem. Soc., 2018, 140, 16124-16133.

24 X. Guan, H. Li, Y. Ma, M. Xue, Q. Fang, Y. Yan, V. Valtchev and S. Qiu, Nat. Chem., 2019, 11, 587-594.

25 E. Jin, M. Asada, Q. Xu, S. Dalapati, M. A. Addicoat, M. A. Brady, H. Xu, T. Nakamura, T. Heine, Q. Chen and D. Jiang, Science, 2017, 357, 673-676.

26 B. Zhang, M. Wei, H. Mao, X. Pei, S. A. Alshmimri, J. A. Reimer and O. M. Yaghi, J. Am. Chem. Soc., 2018, 140, 12715-12719.

27 S. Kandambeth, K. Dey and R. Banerjee, J. Am. Chem. Soc., 2018, 141, 1807-1822.

28 H. Qian, C. Yang and X. Yan, Nat. Commun., 2016, 7, 12104-12110.

29 J. Zhang, X. Han, X. Wu, Y. Liu and Y. Cui, J. Am. Chem. Soc., 2017, 139, 8277-8285.

30 G. Liu, J. Sheng and Y. Zhao, Sci. China: Chem., 2017, 60, 1015-1022.

31 X. Liu, D. Huang, C. Lai, G. Zeng, L. Qin, H. Wang, H. Yi, B. Li, S. Liu and M. Zhang, Chem. Soc. Rev., 2019, 48, 5266-5302.

32 J. L. Segura, S. Royuela and M. M. Ramos, Chem. Soc. Rev., 2019, 48, 3903-3945.

33 H. Wang, Z. Zeng, P. Xu, L. Li, G. Zeng, R. Xiao, Z. Tang, D. Huang, L. Tang and C. Lai, Chem. Soc. Rev., 2019, 48, 488-516.

34 S. Yuan, X. Li, J. Zhu, G. Zhang, P. Van Puyvelde and B. Van der Bruggen, Chem. Soc. Rev., 2019, 48, 2665-2681.

35 H. Vardhan, A. Nafady, A. M. Al-Enizi and S. Ma, Nanoscale, 2019, 11, 21679-21708.

36 J. L. Segura, M. J. Mancheno and F. Zamora, Chem. Soc. Rev., 2016, 45, 5635-5671.

37 R. K. Sharma, P. Yadav, M. Yadav, R. Gupta, P. Rana, A. Srivastava, R. Zbořil, R. S. Varma, M. Antonietti and M. B. Gawande, Mater. Horiz., 2020, 7, 411-454.

38 X. H. Liu, C. Z. Guan, D. Wang and L. J. Wan, Adv. Mater., 2014, 26, 6912-6920.

39 S. S. Han, J. L. Mendoza-Cortés and W. A. Goddard III, Chem. Soc. Rev., 2009, 38, 1460-1476.

40 N. Huang, P. Wang and D. Jiang, Nat. Rev. Mater., 2016, 1, 16068-16085.

41 X. Feng, X. Ding and D. Jiang, Chem. Soc. Rev., 2012, 41, 6010-6022.

42 X. Feng, L. Chen, Y. Dong and D. Jiang, Chem. Commun., 2011, 47, 1979-1981.

43 J. W. Colson, A. R. Woll, A. Mukherjee, M. P. Levendorf, E. L. Spitler, V. B. Shields, M. G. Spencer, J. Park and W. R. Dichtel, Science, 2011, 332, 228-231.

44 A. Halder, M. Ghosh, A. Khayum M, S. Bera, M. Addicoat, H. S. Sasmal, S. Karak, S. Kurungot and R. Banerjee, J. Am. Chem. Soc., 2018, 140, 10941-10945.

45 P. Kuhn, M. Antonietti and A. Thomas, Angew. Chem., Int. Ed., 2008, 47, 3450-3453.

46 H. Ren, T. Ben, E. Wang, X. Jing, M. Xue, B. Liu, Y. Cui, S. Qiu and G. Zhu, Chem. Commun., 2010, 46, 291-293. 
47 M. J. Bojdys, J. Jeromenok, A. Thomas and M. Antonietti, Adv. Mater., 2010, 22, 2202-2205.

48 N. L. Campbell, R. Clowes, L. K. Ritchie and A. I. Cooper, Chem. Mater., 2009, 21, 204-206.

49 L. K. Ritchie, A. Trewin, A. Reguera-Galan, T. Hasell and A. I. Cooper, Microporous Mesoporous Mater., 2010, 132, 132-136.

50 B. P. Biswal, S. Chandra, S. Kandambeth, B. Lukose, T. Heine and R. Banerjee, J. Am. Chem. Soc., 2013, 135, 5328-5331.

51 T. Shiraki, G. Kim and N. Nakashima, Chem. Lett., 2015, 44, 1488-1490.

52 A. de la Peña Ruigómez, D. Rodríguez-San-Miguel, K. C. Stylianou, M. Cavallini, D. Gentili, F. Liscio, S. Milita, O. M. Roscioni, M. L. Ruiz-González and C. Carbonell, Chem. - Eur. J., 2015, 21, 10666-10670.

53 M. Dogru, M. Handloser, F. Auras, T. Kunz, D. Medina, A. Hartschuh, P. Knochel and T. Bein, Angew. Chem., Int. Ed., 2013, 52, 2920-2924.

54 S. Dalapati, E. Jin, M. Addicoat, T. Heine and D. Jiang, J. Am. Chem. Soc., 2016, 138, 5797-5800.

55 S. S. Han, H. Furukawa, O. M. Yaghi and W. A. Goddard III, J. Am. Chem. Soc., 2008, 130, 11580-11581.

56 E. L. Spitler and W. R. Dichtel, Nat. Chem., 2010, 2, 672-677.

57 S. Chandra, S. Kandambeth, B. P. Biswal, B. Lukose, S. M. Kunjir, M. Chaudhary, R. Babarao, T. Heine and R. Banerjee, J. Am. Chem. Soc., 2013, 135, 17853-17861.

58 R. W. Tilford, W. R. Gemmill, H.-C. zur Loye and J. J. Lavigne, Chem. Mater., 2006, 18, 5296-5301.

59 T. Ma, J. Li, J. Niu, L. Zhang, A. S. Etman, C. Lin, D. Shi, P. Chen, L.-H. Li and X. Du, J. Am. Chem. Soc., 2018, 140, 6763-6766.

60 H. Xu, S. Tao and D. Jiang, Nat. Mater., 2016, 15, 722-726.

61 X. Guan, Y. Ma, H. Li, Y. Yusran, M. Xue, Q. Fang, Y. Yan, V. Valtchev and S. Qiu, J. Am. Chem. Soc., 2018, 140, 4494-4498.

62 S. Xu, G. Wang, B. P. Biswal, M. Addicoat, S. Paasch, W. Sheng, X. Zhuang, E. Brunner, T. Heine and R. Berger, Angew. Chem., Int. Ed., 2019, 58, 849-853.

63 S. Dalapati, S. Jin, J. Gao, Y. Xu, A. Nagai and D. Jiang, J. Am. Chem. Soc., 2013, 135, 17310-17313.

64 B. J. Smith and W. R. Dichtel, J. Am. Chem. Soc., 2014, 136, 8783-8789.

65 H. Lyu, C. S. Diercks, C. Zhu and O. M. Yaghi, J. Am. Chem. Soc., 2019, 141, 6848-6852.

66 R. Chen, J. L. Shi, Y. Ma, G. Lin, X. Lang and C. Wang, Angew. Chem., Int. Ed., 2019, 58, 6430-6434.

67 W. Ji, L. Xiao, Y. Ling, C. Ching, M. Matsumoto, R. P. Bisbey, D. E. Helbling and W. R. Dichtel, J. Am. Chem. Soc., 2018, 140, 12677-12681.

68 T. Ma, E. A. Kapustin, S. X. Yin, L. Liang, Z. Zhou, J. Niu, L.-H. Li, Y. Wang, J. Su, J. Li, X. Wang, W. D. Wang, W. Wang, J. Sun and O. M. Yaghi, Science, 2018, 361, 48-52.

69 A. M. Evans, L. R. Parent, N. C. Flanders, R. P. Bisbey, E. Vitaku, M. S. Kirschner, R. D. Schaller, L. X. Chen,
N. C. Gianneschi and W. R. Dichtel, Science, 2018, 361, 52-57.

70 P. L. Llewellyn, G. Maurin, T. Devic, S. Loera-Serna, N. Rosenbach, C. Serre, S. Bourrelly, P. Horcajada, Y. Filinchuk and G. Férey, J. Am. Chem. Soc., 2008, 130, 12808-12814.

71 Y.-B. Zhang, J. Su, H. Furukawa, Y. Yun, F. Gándara, A. Duong, X. Zou and O. M. Yaghi, J. Am. Chem. Soc., 2013, 135, 16336-16339.

72 H. Xu, X. Chen, J. Gao, J. Lin, M. Addicoat, S. Irle and D. Jiang, Chem. Commun., 2014, 50, 1292-1294.

73 H. Xu, J. Gao and D. Jiang, Nat. Chem., 2015, 7, 905-912.

74 S. Zhang, Y. Zheng, H. An, B. Aguila, C. X. Yang, Y. Dong, W. Xie, P. Cheng, Z. Zhang, Y. Chen and S. Ma, Angew. Chem., Int. Ed., 2018, 57, 16754-16759.

75 Q. Sun, C. W. Fu, B. Aguila, J. Perman, S. Wang, H. Y. Huang, F. S. Xiao and S. Ma, J. Am. Chem. Soc., 2018, 140, 984-992.

76 C. Yuan, X. Wu, R. Gao, X. Han, Y. Liu, Y. Long and Y. Cui, J. Am. Chem. Soc., 2019, 141, 20187-20197.

77 H. Xu, S. Ding, W. An, H. Wu and W. Wang, J. Am. Chem. Soc., 2016, 138, 11489-11492.

78 L. Wang, J. Zhou, Y. Lan, S. Ding, W. Yu and W. Wang, Angew. Chem., Int. Ed., 2019, 58, 9443-9447.

79 K. Zhang, S. Cai, Y. Yan, Z. He, H. Lin, X. Huang, S. Zheng, J. Fan and W. Zhang, J. Chromatogr. A, 2017, 1519, 100-109.

80 J. Zhang, X. Han, X. Wu, Y. Liu and Y. Cui, J. Am. Chem. Soc., 2017, 139, 8277-8285.

81 J. Zhang, X. Han, X. Wu, Y. Liu and Y. Cui, ACS Sustainable Chem. Eng., 2019, 7, 5065-5071.

82 H. Ma, J. Kan, G. Chen, C. Chen and Y. Dong, Chem. Mater., 2017, 29, 6518-6524.

83 H. Ma, C. Zhao, G. Chen and Y. Dong, Nat. Commun., 2019, 10, 1-9.

84 X. Wang, X. Han, J. Zhang, X. Wu, Y. Liu and Y. Cui, J. Am. Chem. Soc., 2016, 138, 12332-12335.

85 X. Han, Q. Xia, J. Huang, Y. Liu, C. Tan and Y. Cui, J. Am. Chem. Soc., 2017, 139, 8693-8697.

86 X. Han, J. Huang, C. Yuan, Y. Liu and Y. Cui, J. Am. Chem. Soc., 2018, 140, 892-895.

87 X. Wu, X. Han, Q. Xu, Y. Liu, C. Yuan, S. Yang, Y. Liu, J. Jiang and Y. Cui, J. Am. Chem. Soc., 2019, 141, 7081-7089.

88 X. Han, J. Zhang, J. Huang, X. Wu, D. Yuan, Y. Liu and Y. Cui, Nat. Commun., 2018, 9, 1294.

89 R. Wang, X. Wei and Y. Feng, Chem. - Eur. J., 2018, 24, 10979-10983.

90 Y. Zhang, J. Duan, D. Ma, P. Li, S. Li, H. Li, J. Zhou, X. Ma, X. Feng and B. Wang, Angew. Chem., Int. Ed., 2017, 56, 16313-16317.

91 J. Dong, Y. Liu and Y. Cui, Chem. Commun., 2014, 50, 14949-14952.

92 M. J. Ingleson, J. P. Barrio, J. Bacsa, C. Dickinson, H. Park and M. J. Rosseinsky, Chem. Commun., 2008, 1287-1289.

93 Y. Kang, S. Chen, F. Wang, J. Zhang and X. Bu, Chem. Commun., 2011, 47, 4950-4952. 
94 W. Xi, Y. Liu, Q. Xia, Z. Li and Y. Cui, Chem. - Eur. J., 2015, 21, 12581-12585.

95 L. Ma, C. D. Wu, M. M. Wanderley and W. Lin, Angew. Chem., Int. Ed., 2010, 49, 8244-8248.

96 Y. Liu, Z. Li, G. Yuan, Q. Xia, C. Yuan and Y. Cui, Inorg. Chem., 2016, 55, 12500-12503.

97 Y. Du, K. Mao, P. Kamakoti, P. Ravikovitch, C. Paur, S. Cundy, Q. Li and D. Calabro, Chem. Commun., 2012, 48, 4606-4608.

98 P. J. Waller, Y. S. AlFaraj, C. S. Diercks, N. N. Jarenwattananon and O. M. Yaghi, J. Am. Chem. Soc., 2018, 140, 9099-9103.

99 L. Guo, S. Jia, C. S. Diercks, X. Yang, S. A. Alshmimri and O. M. Yaghi, Angew. Chem., Int. Ed., 2020, 59, 2023-2027.

100 C. Bi, X. Zheng, S. Azaria, S. Beeram, Z. Li and D. S. Hage, Separations, 2016, 3, 27-52.

101 A. L. Margolin, Enzyme Microb. Technol., 1993, 15, 266-280. 102 S. Pizzarello and A. L. Weber, Science, 2004, 303, 1151.

103 S. Li and W. C. Purdy, Chem. Rev., 1992, 92, 1457-1470.

104 K. Uekama, F. Hirayama and T. Irie, Chem. Rev., 1998, 98, 2045-2076.

105 G. Wenz, B. Han and A. Müller, Chem. Rev., 2006, 106, 782-817.

106 D. J. Tranchemontagne, Z. Ni, M. O'Keeffe and O. M. Yaghi, Angew. Chem., Int. Ed., 2008, 47, 5136-5147.

107 J. R. Long and O. M. Yaghi, Chem. Soc. Rev., 2009, 38, 1213-1214.

108 M. O’Keeffe, M. A. Peskov, S. J. Ramsden and O. M. Yaghi, Acc. Chem. Res., 2008, 41, 1782-1789.

109 D. Seebach, A. K. Beck and A. Heckel, Angew. Chem., Int. Ed., 2001, 40, 92-138.

110 X. Wang, J. Zhang, Y. Liu and Y. Cui, Bull. Chem. Soc. Jpn., 2014, 87, 435-440.

111 H. Teller, M. Corbet, L. Mantilli, G. Gopakumar, R. Goddard, W. Thiel and A. Fürstner, J. Am. Chem. Soc., 2012, 134, 15331-15342.

112 Q. Xia, Z. Li, C. Tan, Y. Liu, W. Gong and Y. Cui, J. Am. Chem. Soc., 2017, 139, 8259-8266.

113 C. Tan, J. Jiao, Z. Li, Y. Liu, X. Han and Y. Cui, Angew. Chem., Int. Ed., 2018, 57, 2085-2090.

114 L. Canali and D. C. Sherrington, Chem. Soc. Rev., 1999, 28, 85-93.

115 C. J. Whiteoak, G. Salassa and A. W. Kleij, Chem. Soc. Rev., 2012, 41, 622-631.

116 G. Li, W. Yu, J. Ni, T. Liu, Y. Liu, E. Sheng and Y. Cui, Angew. Chem., Int. Ed., 2008, 47, 1245-1249.

117 C. Zhu, G. Yuan, X. Chen, Z. Yang and Y. Cui, J. Am. Chem. Soc., 2012, 134, 8058-8061.

118 J. F. Larrow and E. N. Jacobsen, Organometallics in Process Chemistry, Springer Berlin Heidelberg, Berlin, Heidelberg, 2004, pp. 123-152.

119 M. S. Sigman and E. N. Jacobsen, J. Am. Chem. Soc., 1998, 120, 5315-5316.

120 C. Baleizao and H. Garcia, Chem. Rev., 2006, 106, 3987-4043.

121 L. Li, X. Feng, X. Cui, Y. Ma, S. Ding and W. Wang, J. Am. Chem. Soc., 2017, 139, 6042-6045.
122 H. Li, X. Feng, P. Shao, J. Chen, C. Li, S. Jayakumar and Q. Yang, J. Mater. Chem. A, 2019, 7, 5482-5492.

123 Y. Cui, H. L. Ngo, P. S. White and W. Lin, Chem. Commun., 2002, 1666-1667.

124 M. Shibasaki and S. Matsunaga, Chem. Soc. Rev., 2006, 35, 269-279.

125 J. M. Brunel, Chem. Rev., 2005, 105, 857-898.

126 L. Ma, C. Abney and W. Lin, Chem. Soc. Rev., 2009, 38, 1248-1256.

127 H. M. El-Kaderi, J. R. Hunt, J. L. Mendoza-Cortés, A. P. Côté, R. E. Taylor, M. O'Keeffe and O. M. Yaghi, Science, 2007, 316, 268-272.

128 G. Lin, H. Ding, R. Chen, Z. Peng, B. Wang and C. Wang, J. Am. Chem. Soc., 2017, 139, 8705-8709.

129 Q. Fang, S. Gu, J. Zheng, Z. Zhuang, S. Qiu and Y. Yan, Angew. Chem., Int. Ed., 2014, 53, 2878-2882.

130 K. Y. Bliokh and F. Nori, Phys. Rev. A: At., Mol., Opt. Phys., 2011, 83, 021803.

131 J. R. Brandt, F. Salerno and M. J. Fuchter, Nat. Rev. Chem., 2017, 1, 0045.

132 R. S. Cahn, C. Ingold and V. Prelog, Angew. Chem., Int. Ed. Engl., 1966, 5, 385-415.

133 L. Ascherl, T. Sick, J. T. Margraf, S. H. Lapidus, M. Calik, C. Hettstedt, K. Karaghiosoff, M. Döblinger, T. Clark and K. W. Chapman, Nat. Chem., 2016, 8, 310-316.

134 Z. Lin, A. M. Slawin and R. E. Morris, J. Am. Chem. Soc., 2007, 129, 4880-4881.

135 S. Zhang, D. Li, D. Guo, H. Zhang, W. Shi, P. Cheng, L. Wojtas and M. J. Zaworotko, J. Am. Chem. Soc., 2015, 137, 15406-15409.

136 J. D. Evans and F. Coudert, J. Am. Chem. Soc., 2016, 138, 6131-6134.

137 D. Dang, P. Wu, C. He, Z. Xie and C. Duan, J. Am. Chem. Soc., 2010, 132, 14321-14323.

138 J. Liu, L. Chen, H. Cui, J. Zhang, L. Zhang and C. Su, Chem. Soc. Rev., 2014, 43, 6011-6061.

139 C. Wu, A. Hu, L. Zhang and W. Lin, J. Am. Chem. Soc., 2005, 127, 8940-8941.

140 M. Yoon, R. Srirambalaji and K. Kim, Chem. Rev., 2011, 112, 1196-1231.

141 T. Lu, W. Yan and R. Xu, Inorg. Chem. Front., 2019, 6, 1938-1951.

142 C. Song and S. Lee, Chem. Rev., 2002, 102, 3495-3524.

143 W. Gong, D. Chu, H. Jiang, X. Chen, Y. Cui and Y. Liu, Nat. Commun., 2019, 10, 600.

144 J. Jiao, Z. Li, Z. Qiao, X. Li, Y. Liu, J. Dong, J. Jiang and Y. Cui, Nat. Commun., 2018, 9, 4423.

145 J. Jiao, C. Tan, Z. Li, Y. Liu, X. Han and Y. Cui, J. Am. Chem. Soc., 2018, 140, 2251-2259.

146 D. Jiang, X. Chen, K. Geng, R. Liu, K. T. Tan, Y. Gong, Z. Li, S. Tao and Q. Jiang, Angew. Chem., Int. Ed., 2020, 59, 5050-5091.

147 P. Pachfule, A. Acharjya, J. Roeser, T. Langenhahn, M. Schwarze, R. Schomäcker, A. Thomas and J. Schmidt, J. Am. Chem. Soc., 2018, 140, 1423-1427.

148 C. Y. Lin, L. Zhang, Z. Zhao and Z. Xia, Adv. Mater., 2017, 29, 1606635. 
149 S. Ding, J. Gao, Q. Wang, Y. Zhang, W. Song, C. Su and W. Wang, J. Am. Chem. Soc., 2011, 133, 19816-19822.

150 L. Stegbauer, K. Schwinghammer and B. V. Lotsch, Chem. Sci., 2014, 5, 2789-2793.

151 C. S. Diercks, S. Lin, N. Kornienko, E. A. Kapustin, E. M. Nichols, C. Zhu, Y. Zhao, C. J. Chang and O. M. Yaghi, J. Am. Chem. Soc., 2018, 140, 1116-1122.

152 P. G. Cozzi, Chem. Soc. Rev., 2004, 33, 410-421.

153 T. P. Yoon and E. N. Jacobsen, Science, 2003, 299, 1691-1693.

154 H. Yang, L. Zhang, L. Zhong, Q. Yang and C. Li, Angew. Chem., Int. Ed., 2007, 46, 6861-6865.

155 Y. Xie, T. Wang, X. Liu, K. Zou and W. Deng, Nat. Commun., 2013, 4, 1960.

156 Z. Yang, C. Zhu, Z. Li, Y. Liu, G. Liu and Y. Cui, Chem. Commun., 2014, 50, 8775-8778.

157 F. Song, C. Wang and W. Lin, Chem. Commun., 2011, 47, 8256-8258.

158 Q. Sun, C. Fu, B. Aguila, J. Perman, S. Wang, H. Huang, F. Xiao and S. Ma, J. Am. Chem. Soc., 2018, 140, 984-992.

159 X. Wu, X. Han, J. Zhang, H. Jiang, B. Hou, Y. Liu and Y. Cui, Organometallics, 2019, 38, 3474-3479.

160 H. Fan, A. Mundstock, J. Gu, H. Meng and J. Caro, J. Mater. Chem. A, 2018, 6, 16849-16853.

161 W. Zhang, L. Zhang, H. Zhao, B. Li and H. Ma, J. Mater. Chem. A, 2018, 6, 13331-13339.

162 D. B. Shinde, G. Sheng, X. Li, M. Ostwal, A. Emwas, K. Huang and Z. Lai, J. Am. Chem. Soc., 2018, 140, 14342-14349.

163 H. Yang, H. Wu, Z. Yao, B. Shi, Z. Xu, X. Cheng, F. Pan, G. Liu, Z. Jiang and X. Cao, J. Mater. Chem. A, 2018, 6, 583-591.

164 K. Dey, M. Pal, K. C. Rout, S. Kunjattu H, A. Das, R. Mukherjee, U. K. Kharul and R. Banerjee, J. Am. Chem. Soc., 2017, 139, 13083-13091.

165 H. Fan, J. Gu, H. Meng, A. Knebel and J. Caro, Angew. Chem., Int. Ed., 2018, 57, 4083-4087.

166 J. Fu, S. Das, G. Xing, T. Ben, V. Valtchev and S. Qiu, J. Am. Chem. Soc., 2016, 138, 7673-7680.

167 X. Kuang, Y. Ma, H. Su, J. Zhang, Y. Dong and B. Tang, Anal. Chem., 2014, 86, 1277-1281.

168 N. M. Maier, P. Franco and W. Lindner, J. Chromatogr. A, 2001, 906, 3-33.

169 T. J. Ward and K. D. Ward, Anal. Chem., 2011, 84, 626-635.
170 G. Gübitz and M. G. Schmid, Biopharm. Drug Dispos., 2001, 22, 291-336.

171 S. A. Wren and R. C. Rowe, J. Chromatogr. A, 1992, 603, 235-241.

172 C. Yamamoto and Y. Okamoto, Bull. Chem. Soc. Jpn., 2004, 77, 227-257.

173 S. Xie, Z. Zhang, Z. Wang and L. Yuan, J. Am. Chem. Soc., 2011, 133, 11892-11895.

174 A. Kewley, A. Stephenson, L. Chen, M. E. Briggs, T. Hasell and A. I. Cooper, Chem. Mater., 2015, 27, 3207-3210.

175 J. Huang, X. Han, S. Yang, Y. Cao, C. Yuan, Y. Liu, J. Wang and Y. Cui, J. Am. Chem. Soc., 2019, 141, 8996-9003.

176 L. Liu, C. Yang and X. Yan, J. Chromatogr. A, 2017, 1479, 137-144.

177 W. P. Lustig, S. Mukherjee, N. D. Rudd, A. V. Desai, J. Li and S. K. Ghosh, Chem. Soc. Rev., 2017, 46, 3242-3285.

178 G. Das, B. P. Biswal, S. Kandambeth, V. Venkatesh, G. Kaur, M. Addicoat, T. Heine, S. Verma and R. Banerjee, Chem. Sci., 2015, 6, 3931-3939.

179 W. Huang, Y. Jiang, X. Li, X. Li, J. Wang, Q. Wu and X. Liu, ACS Appl. Mater. Interfaces, 2013, 5, 8845-8849.

180 Y. Zhang, X. Shen, X. Feng, H. Xia, Y. Mu and X. Liu, Chem. Commun., 2016, 52, 11088-11091.

181 W. Li, C. Yang and X. Yan, Chem. Commun., 2017, 53, 11469-11471.

182 Y. Yang, Z. Zhao, Y. Yang, G. Li and C. Hao, New J. Chem., 2019, 43, 9274-9279.

183 Z. Zhou, W. Zhong, K. Cui, Z. Zhuang, L. Li, L. Li, J. Bi and Y. Yu, Chem. Commun., 2018, 54, 9977-9980.

184 S. Ding, M. Dong, Y. Wang, Y. Chen, H. Wang, C. Su and W. Wang, J. Am. Chem. Soc., 2016, 138, 3031-3037.

185 G. A. Hembury, V. V. Borovkov and Y. Inoue, Chem. Rev., 2008, 108, 1-73.

186 L. Friedman and J. G. Miller, Science, 1971, 172, 1044-1046.

187 C. Dekker, Nat. Nanotechnol., 2007, 2, 209-215.

188 Z. Zhu, D. Wang, Y. Tian and L. Jiang, J. Am. Chem. Soc., 2019, 141, 8658-8669.

189 Y. Ying, Y. Hu, R. Gao, R. Yu, Z. Gu, L. Lee and Y. Long, J. Am. Chem. Soc., 2018, 140, 5385-5392.

190 X. Ding, L. Chen, Y. Honsho, X. Feng, O. Saengsawang, J. Guo, A. Saeki, S. Seki, S. Irle and S. Nagase, J. Am. Chem. Soc., 2011, 133, 14510-14513.

191 N. Huang, X. Chen, R. Krishna and D. Jiang, Angew. Chem., Int. Ed., 2015, 54, 2986-2990.

192 H. Xu and D. Jiang, Nat. Chem., 2014, 6, 564-566. 